\title{
Approximation Results for Discontinuous Games with an Application to Equilibrium Refinement*
}

\author{
Oriol Carbonell-Nicolau ${ }^{\dagger} \quad$ Richard P. McLean ${ }^{\ddagger}$
}

August 2012

\begin{abstract}
We provide approximation results for Nash equilibria in possibly discontinuous games when payoffs and strategy sets are perturbed. We then prove existence results for a new "finitistic" infinite-game generalization of Selten's [24] notion of perfection, and study some of its properties. The existence results, which rely on the approximation theorems, relate existing notions of perfection to the new specification.

Keywords: infinite normal-form game, discontinuous game, Nash equilibrium correspondence, better-reply security, equilibrium refinement, finite approximation, trembling-hand perfect equilibrium, limit-of-finite perfect equilibrium.

JEL classification: C72.
\end{abstract}

\section{Introduction}

In this paper, we provide approximation results for Nash equilibria in possibly discontinuous games when payoffs and strategy sets are perturbed. These

*We thank two anonymous referees for helpful comments.

${ }^{\dagger}$ Department of Economics, Rutgers University, 75 Hamilton Street, New Brunswick, NJ 08901, USA. E-mail: carbonell@econ.rutgers.edu.

$\ddagger$ Department of Economics, Rutgers University, 75 Hamilton Street, New Brunswick, NJ 08901, USA. E-mail: rpmclean@rci.rutgers.edu. 
conditions are then used to derive existence results for a new "finitistic" infinite-game generalization of Selten's [24] notion of perfection. Some of the properties of the new refinement specification are studied and related to existing formulations of perfection. To introduce the issues that we address, suppose that $G=\left(X_{i}, u_{i}\right)_{i=1}^{N}$ is an $N$-player strategic-form game defined by action sets $X_{i}$ and payoff functions $u_{i}$.

We begin by identifying general conditions under which an approximation result of the following type will hold:

\section{Statement C. If}

(i) for each $i,\left(X_{i}^{\alpha}\right)$ is a net of subsets of $X_{i}$ and $\left(u_{i}^{\alpha}\right)$ is a net in the space of payoff functions defined on $X:=\times_{i=1}^{N} X_{i}$ with limit $u_{i}$,

(ii) $\left(x^{\alpha}\right)$ is a net in $X$ with limit $x \in X$ such that, for each $\alpha, x^{\alpha}$ is an $\varepsilon^{\alpha}$-Nash equilibrium of the game $G^{\alpha}=\left(X_{i}^{\alpha}, u_{i}^{\alpha}\right)_{i=1}^{N}$, and

(iii) $\varepsilon^{\alpha} \rightarrow 0$,

then $x$ is a Nash equilibrium of the game $G=\left(X_{i}, u_{i}\right)_{i=1}^{N}$.

This approximation result and some variants of it will be useful when addressing questions of equilibrium refinement. The archetype for all results of this kind is the classic closed graph theorem for the Nash equilibrium correspondence of the mixed extension of a game when the payoff functions are the parameters. This classic result relies on continuity of the payoff functions. Several papers have addressed the more general approximation question of Statement $\mathrm{C}$ above in the framework of continuous and discontinuous games. ${ }^{1}$

We introduce the notion of limit better-reply security of a game $G=$ $\left(X_{i}, u_{i}\right)_{i=1}^{N}$ with respect to a net $\left(X^{\alpha}, u^{\alpha}\right)$, where $\left(X^{\alpha}\right)$ is a net of subsets of $X$ and $\left(u^{\alpha}\right)$ is an approximating net for $\left(u_{1}, \ldots, u_{N}\right)$. We show that this condition is, essentially, a reformulation of Statement C. If $X^{\alpha}=X$ and $u^{\alpha}=u$, then we say that $G=\left(X_{i}, u_{i}\right)_{i=1}^{N}$ satisfies limit better-reply security. Limit better-reply security is weaker than Barelli and Soza's [6] generalized better-reply security, which is, in turn, weaker than the notion of better-reply

\footnotetext{
${ }^{1}$ See Lucchetti and Patrone [19], Gürkan and Pang [17], and Bagh [4]. The reader is referred to Carbonell-Nicolau and McLean [12] for a detailed comparison between our work and these references.
} 
security introduced in Reny's [22] work. ${ }^{2}$ The reader is referred to CarbonellNicolau and McLean [12] for a detailed comparison between limit better-reply security and related notions introduced by several authors. ${ }^{3}$

In Section 4, we provide appropriate analogues for our definitions for the mixed extension of a game, and extend the approximation results to the case of mixed strategies.

Section 5 applies the approximation results derived in Sections 3 and 4 to the analysis of perfect equilibrium in discontinuous games. We briefly survey the existing infinite-game extensions of perfection, including Simon and Stinchcombe's [26] limit-of-finite formulations. The limit-of-finite approach takes the view that infinite models are merely convenient representations of "true" models, which are large but finite. Simon and Stinchcombe [26] define an $\varepsilon$-perfect equilibrium as a completely mixed $\varepsilon$-equilibrium (where the players choose actions that are in some sense close to their set of best responses to the other players' strategies), and a limit-of-finite (lof) perfect equilibrium as the limit of $\varepsilon$-perfect equilibria for successively larger finite approximations of an infinite game. It is shown in [26] that the notion of lof perfection is ill-suited even in continuous games, for it fails a weakening of admissibility, termed limit admissibility in [26], which requires that no player choose an action in the interior of the set of weakly dominated actions.

We introduce a new limit-of-finite approach, in the spirit of Simon and Stinchcombe's [26] formulation, that does not suffer from this drawback. This approach relies on finite approximations to Selten perturbations of a game. A Selten perturbation may be viewed as a model of slight mistakes in which any player may tremble and play any one of her actions. Standard notions of perfection, when stated in terms of Selten perturbations, define a perfect equilibrium as the limit of some sequence of (exact) equilibria of neighboring Selten perturbations of a game. Thus, an equilibrium $\mu$ is perfect if there exists a sequence of models of (low-probability) mistakes that have at least one equilibrium close to $\mu$, so that $\mu$ describes approximate equilibrium behavior when the players interact in the perturbed game. Our "finitistic" approach to perfection defines a limit-of-finite perfect equilibrium as the limit of sequences of (exact) equilibria of neighboring finite Selten per-

\footnotetext{
${ }^{2}$ For earlier work on discontinuous games, see Dasgupta and Maskin [16], Simon [25], and Lebrun[18].

${ }^{3}$ See, for instance, Dasgupta and Maskin [16], Simon [25], Reny [22, 23], Bagh and Jofre [3], Carmona [13, 14], de Castro [15], Prokopovych [21], Barelli and Soza [6], and McLennan et al. [20].
} 
turbations that respect the strategic aspects of the original (infinite) game, in the sense that they can be interpreted as "true" representations of certain infinite Selten perturbations. The consistency between the finite and the infinite models is obtained by requiring that an equilibrium be the limit of some set of sequences of equilibria of finite Selten perturbations, where the set of sequences is sufficiently close to some "mirror" sequence of infinite perturbations. An equilibrium with this property is called a strong limit-offinite (lof) perfect equilibrium. Strong lof perfect equilibria are lof perfect in Simon and Stinchcombe's [26] sense (Proposition 1), but the converse is not true.

Subsection 5.1 provides existence results for strong lof perfect equilibrium profiles. We first state and prove a result relating the convergence theorems furnished in Sections 3 and 4 to the existence of strong lof perfect and trembling-hand perfect equilibria in discontinuous games (Proposition 2 ). This result is then used to prove existence results in terms of the primitives of a game, i.e., the game's payoff functions defined over pure strategies (Theorems 5 and 6). Our main existence result, Theorem 6, states that compact games satisfying generic entire payoff security, generic local equi-upper semicontinuity, and upper semicontinuity of the sum of payoffs possess strong lof perfect equilibria that are trembling-hand perfect. Generic entire payoff security and generic local equi-upper semicontinuity are easy to verify in a variety of economic games ( $c f$. Carbonell-Nicolau $[9,11]$ ).

Subsection 5.2 studies the relationship between strong lof perfection and limit admissibility. For continuous games, all strong lof perfect equilibria are trembling-hand perfect (Theorem 7 ). This result can be combined with results from Simon and Stinchcombe [26] to conclude that strong lof perfection satisfies (unlike Simon and Stinchcombe's [26] lof perfection) limit admissibility in continuous games (Theorem 8).

\section{Preliminaries}

A strategic-form game is a collection $G=\left(X_{i}, u_{i}\right)_{i=1}^{N}$, where $N$ is a finite number of players, $X_{i}$ is a nonempty set of actions for player $i$, and $u_{i} \in B(X)$, where $B(X)$ denotes the space of bounded, real-valued functions defined on $X:=\times_{i=1}^{N} X_{i}$. We view $B(X)$ as a metric space with associated metric defined by

$$
\rho(f, g):=\sup _{x \in X}|f(x)-g(x)|
$$


Let $X_{-i}:=\times_{j \neq i} X_{j}$ for each $i$. We will often abuse notation and simply write $G=\left(X_{i}, u_{i}\right)$ for $G=\left(X_{i}, u_{i}\right)_{i=1}^{N}$. Given $i$ and $\left(x_{i}, x_{-i}\right) \in X_{i} \times X_{-i}$, we employ the standard convention and write $\left(x_{1}, \ldots, x_{N}\right)$ in $X$ as $\left(x_{i}, x_{-i}\right)$. If $G=\left(X_{i}, u_{i}\right)$ is a game and $Y_{i} \subseteq X_{i}$ for each $i$, we will write $\left(Y_{i},\left.u_{i}\right|_{Y_{i}}\right)_{i=1}^{N}$ simply as $\left(Y_{i}, u_{i}\right)_{i=1}^{N}$ or $\left(Y_{i}, u_{i}\right)$.

Let $U(X)$ denote cartesian product of $N$ copies of $B(X)$. We also view $U(X)$ as a metric space, and denote, by a slight abuse of notation, the associated metric again by $\rho$, i.e.,

$$
\rho\left(\left(f_{1}, \ldots, f_{N}\right),\left(g_{1}, \ldots, g_{N}\right)\right):=\max _{i \in\{1, \ldots, N\}}\left[\sup _{x \in X}\left|f_{i}(x)-g_{i}(x)\right|\right] .
$$

Consequently, a net $\left(u^{\alpha}\right)$ in $U(X)$ is convergent with limit $u$ if and only if for each $i$, the net $\left(u_{i}^{\alpha}\right)$ is uniformly convergent with limit $u_{i}$.

If $G=\left(X_{i}, u_{i}\right)$ is a game then a test net for $G$ is a net $\left(G^{\nu}\right)=\left(X_{i}^{\nu}, u_{i}^{\nu}\right)$, where, for each $i,\left(X_{i}^{\nu}\right)$ is a net of nonempty subsets of $X_{i}$ and $\left(u_{i}^{\nu}\right)$ is a net of functions in $B(X)$. Note that we do not (yet) assume that $\left(u_{i}^{\nu}\right)$ is an approximating net for $u_{i}$.

Definition 1. If $\varepsilon \geq 0$, then a strategy profile $x=\left(x_{i}, x_{-i}\right)$ in $X$ is an $\varepsilon$ Nash equilibrium of $G=\left(X_{i}, u_{i}\right)$ if $u_{i}\left(y_{i}, x_{-i}\right) \leq u_{i}(x)+\varepsilon$ for each $y_{i} \in X_{i}$ and every $i$. A 0-Nash equilibrium will be called a Nash equilibrium.

Define a correspondence $\mathcal{N}_{X}: U(X) \rightrightarrows X$ that assigns to each profile $u=\left(u_{1}, \ldots, u_{N}\right) \in U(X)$ the set of Nash equilibria $\mathcal{N}_{X}(u)$ of $\left(X_{i}, u_{i}\right)$. Given $\varepsilon \geq 0$, define a correspondence $\mathcal{N}_{X}^{\varepsilon}: U(X) \rightrightarrows X$ that assigns to each profile $u=\left(u_{1}, . ., u_{N}\right) \in U(X)$ the set of $\varepsilon$-Nash equilibria $\mathcal{N}_{X}^{\varepsilon}(u)$ of $\left(X_{i}, u_{i}\right)$.

When each $X_{i}$ is a (nonempty) topological space, $G=\left(X_{i}, u_{i}\right)_{i=1}^{N}$ is called a topological game. When each $X_{i}$ is a nonempty metric space, we say that $G$ is a metric game. If, in addition each $X_{i}$ is a compact topological (metric) space, then $G$ is called a compact topological (metric) game.

If $G$ is a topological game, we are not (yet) assuming that the strategy spaces $X_{i}$ are first countable (or Hausdorff for that matter). Consequently, our approximation results in this section are formulated in terms of nets. However, virtually all of our results will hold if we replace "net" and "subnet" with "sequence" and "subsequence." 4 If we were to work only in a metric space framework, then we could have used sequences everywhere and in fact,

\footnotetext{
${ }^{4}$ Some care is required here. For example, the closedness conclusion of Corollary 1 would not hold if we presented our definitions in terms of sequences rather than nets.
} 
we explicitly use sequences in Conditions 5-6, Theorem 4, and Corollary 3 below. Throughout the paper, we will use $\left(x^{\alpha}\right)=\left(x^{\alpha}\right)_{\alpha \in D}$ to denote a net with indices belonging to a directed set $(D, \prec)$. As we stated in the introduction, one of our main goals is to identify general conditions under which an approximation result like that posed in Statement $\mathrm{C}$ will hold. As we show below, results of this type will be useful in the study of refinements of equilibrium.

If $\left(S^{\alpha}\right)$ is a net of subsets of a topological space $S$, define the (PainleveKuratowski) topological limit superior of $\left(S^{\alpha}\right)$, denoted $\operatorname{Ls}\left(S^{\alpha}\right)$, to be the set of $y \in S$ such that there exist a subnet $\left(S^{\beta}\right)$ and a net $\left(y^{\beta}\right)$ satisfying $y^{\beta} \in S^{\beta}$ for each $\beta$ and $y^{\beta} \rightarrow y$. Define the topological limit inferior of $\left(S^{\alpha}\right)$, denoted $\operatorname{Li}\left(S^{\alpha}\right)$, to be the set of $y \in S$ such that there exists a net $\left(y^{\alpha}\right)$ satisfying $y^{\alpha} \in S^{\alpha}$ for each $\alpha$ and $y^{\alpha} \rightarrow y$.

\section{Approximation results for pure-strategy Nash equilibria of topological games}

Condition 1. Let $G=\left(X_{i}, u_{i}\right)$ be a topological game. Suppose that $\left(X_{i}^{\nu}, u_{i}^{\nu}\right)$ is a test net for $G$. The game $\left(X_{i}, u_{i}\right)$ satisfies limit better-reply security with respect to $\left(X_{i}^{\nu}, u_{i}^{\nu}\right)$ if the following condition is satisfied: if $\left(X_{i}^{\alpha}, u_{i}^{\alpha}\right)$ is a subnet of $\left(X_{i}^{\nu}, u_{i}^{\nu}\right)$, if $\left(x^{\alpha}, u^{\alpha}\left(x^{\alpha}\right)\right) \in X \times \mathbb{R}^{N}$ is a convergent net with limit $(x, \gamma) \in X \times \mathbb{R}^{N}$ satisfying $x^{\alpha} \in X^{\alpha}$ for each $\alpha$, and if $x$ is not a Nash equilibrium of $\left(X_{i}, u_{i}\right)$, then there exist an $i$, an $\eta>\gamma_{i}$, a subnet $\left(x^{\beta}\right)$ of $\left(x^{\alpha}\right)$ and a net $\left(y_{i}^{\beta}\right)$ such that, for each $\beta, y_{i}^{\beta} \in X_{i}^{\beta}$ and $u_{i}^{\beta}\left(y_{i}^{\beta}, x_{-i}^{\beta}\right) \geq \eta$.

Condition 2. A topological game $G=\left(X_{i}, u_{i}\right)$ satisfies limit better-reply security if and only if $G$ satisfies limit better-reply security with respect to $\left(X_{i}^{\nu}, u_{i}^{\nu}\right)$, with $\left(X_{i}^{\nu}, u_{i}^{\nu}\right)=\left(X_{i}, u_{i}\right)$ for all $\nu$. That is, $G$ satisfies limit betterreply security if the following holds: if $\left(x^{\alpha}, u\left(x^{\alpha}\right)\right) \in X \times \mathbb{R}^{N}$ is a convergent net with limit $(x, \gamma) \in X \times \mathbb{R}^{N}$ and if $x$ is not a Nash equilibrium of $\left(X_{i}, u_{i}\right)$, then there exist an $i$, an $\eta>\gamma_{i}$, a subnet $\left(x^{\beta}\right)$ of $\left(x^{\alpha}\right)$ and a net $\left(y_{i}^{\beta}\right)$ such that, for each $\beta, y_{i}^{\beta} \in X_{i}$ and $u_{i}\left(y_{i}^{\beta}, x_{-i}^{\beta}\right) \geq \eta$.

Theorem 1. Suppose that $G=\left(X_{i}, u_{i}\right)$ is a topological game and suppose that $\left(X_{i}^{\nu}, u_{i}^{\nu}\right)$ is a test net for $G$. The following are equivalent:

(1) $G=\left(X_{i}, u_{i}\right)$ satisfies limit better-reply security with respect to $\left(X_{i}^{\nu}, u_{i}^{\nu}\right)$ (Condition 1). 
(2) If $\left(X_{i}^{\alpha}, u_{i}^{\alpha}\right)$ is a subnet of $\left(X_{i}^{\nu}, u_{i}^{\nu}\right)$ and if $\left(x^{\alpha}, u^{\alpha}\left(x^{\alpha}\right)\right)$ is a convergent net with limit $(x, \gamma) \in X \times \mathbb{R}^{N}$ satisfying $x^{\alpha} \in \mathcal{N}_{X^{\alpha}}^{\varepsilon^{\alpha}}\left(u^{\alpha}\right)$ for each $\alpha$, where $\varepsilon^{\alpha} \rightarrow 0$, then $x \in \mathcal{N}_{X}(u)$.

Proof. Suppose that (1) holds. Let $\left(X_{i}^{\alpha}, u_{i}^{\alpha}\right)$ be a subnet of $\left(X_{i}^{\nu}, u_{i}^{\nu}\right)$. Suppose that $\left(x^{\alpha}, u^{\alpha}\left(x^{\alpha}\right)\right)$ is a convergent net with limit $(x, \gamma) \in X \times \mathbb{R}^{N}$ satisfying $x^{\alpha} \in \mathcal{N}_{X^{\alpha}}^{\varepsilon^{\alpha}}\left(u^{\alpha}\right)$ for each $\alpha$, where $\varepsilon^{\alpha} \rightarrow 0$. If $x$ is not a Nash equilibrium of $\left(X_{i}, u_{i}\right)$, then there exist an $i$, an $\eta>\gamma_{i}$, a subnet $\left(x^{\beta}\right)$ of $\left(x^{\alpha}\right)$ and a net $\left(y_{i}^{\beta}\right)$ such that, for each $\beta, y_{i}^{\beta} \in X_{i}^{\beta}$ and $u_{i}^{\beta}\left(y_{i}^{\beta}, x_{-i}^{\beta}\right) \geq \eta$. Now choose $\varepsilon$ and an index $\beta^{\prime}$ so that $\eta>\gamma_{i}+\varepsilon, \frac{\varepsilon}{2}>\varepsilon^{\beta^{\prime}}$, and $u_{i}^{\beta^{\prime}}\left(x_{i}^{\beta^{\prime}}, x_{-i}^{\beta^{\prime}}\right)<\gamma_{i}+\frac{\varepsilon}{2}$. Then

$$
u_{i}^{\beta^{\prime}}\left(y_{i}^{\beta^{\prime}}, x_{-i}^{\beta^{\prime}}\right) \geq \eta>\gamma_{i}+\varepsilon>u_{i}^{\beta^{\prime}}\left(x_{i}^{\beta^{\prime}}, x_{-i}^{\beta^{\prime}}\right)+\varepsilon^{\beta^{\prime}},
$$

contradicting the assumption that $x^{\beta^{\prime}} \in \mathcal{N}_{X^{\beta^{\prime}}}^{\varepsilon^{\beta^{\prime}}}\left(u^{\beta^{\prime}}\right)$. This establishes (2).

Now suppose that (2) holds. ${ }^{5}$ Suppose $\left(X_{i}^{\alpha}, u_{i}^{\alpha}\right)$ is a subnet of $\left(X_{i}^{\nu}, u_{i}^{\nu}\right)$, $\left(x^{\alpha}, u^{\alpha}\left(x^{\alpha}\right)\right) \in X \times \mathbb{R}^{N}$ is a convergent net with limit $(x, \gamma) \in X \times \mathbb{R}^{N}$ satisfying $x^{\alpha} \in X^{\alpha}$ for each $\alpha$, and $x$ is not a Nash equilibrium of $\left(X_{i}, u_{i}\right)$.

For each $\alpha$ and $i$, define

$$
v_{i}^{\alpha}\left(x^{\alpha}\right):=\sup _{\xi_{i} \in X_{i}^{\alpha}} u_{i}^{\alpha}\left(\xi_{i}, x_{-i}^{\alpha}\right)
$$

and let

$$
\varepsilon^{\alpha}:=\max _{i \in\{1, \ldots, N\}}\left[v_{i}^{\alpha}\left(x^{\alpha}\right)-u_{i}^{\alpha}\left(x^{\alpha}\right)\right] .
$$

Note that $0 \leq v_{i}^{\alpha}\left(x^{\alpha}\right)-u_{i}^{\alpha}\left(x^{\alpha}\right) \leq \varepsilon^{\alpha}$ for each $i$ implies that $x^{\alpha} \in \mathcal{N}_{X^{\alpha}}^{\varepsilon^{\alpha}}\left(u^{\alpha}\right)$. Since $x$ is not a Nash equilibrium of $\left(X_{i}, u_{i}\right)$, it follows from (2) that the net $\left(\varepsilon^{\alpha}\right)$ is not convergent with limit 0 . Consequently, there exists an $\varepsilon>0$ and subnets $\left(\varepsilon^{\lambda}\right)$ and $\left(x^{\lambda}\right)$ satisfying the following conditions for each $\lambda$ : $\varepsilon^{\lambda} \geq \varepsilon$ and $u_{j}^{\lambda}\left(x^{\lambda}\right)>\gamma_{j}-\frac{\varepsilon}{4}$ for each $j$ and there exists a player $i$ for whom $v_{i}^{\lambda}\left(x^{\lambda}\right) \geq u_{i}^{\lambda}\left(x^{\lambda}\right)+\varepsilon$. Since the player set is finite, it follows that there exist an $i$, a subnet $\left(x^{\beta}\right)$ of $\left(x^{\lambda}\right)$, and a net $\left(y_{i}^{\beta}\right)$ satisfying $y_{i}^{\beta} \in X_{i}^{\beta}$ for each $\beta$ and

$$
u_{i}^{\beta}\left(y_{i}^{\beta}, x_{-i}^{\beta}\right)>u_{i}^{\beta}\left(x^{\beta}\right)+\frac{\varepsilon}{2}>\gamma_{i}+\frac{\varepsilon}{4}=\eta,
$$

as desired.

Theorem 1 characterizes a stability property for Nash equilibria in discontinuous games in which $\left(X_{i}^{\nu}, u_{i}^{\nu}\right)$ is an approximating net for the game

\footnotetext{
${ }^{5}$ We thank an anonymous referee for suggesting this argument.
} 
$G=\left(X_{i}, u_{i}\right)$ with respect to which $G$ satisfies limit better-reply security. If $\left(x^{\alpha}\right)$ is a convergent sequence of $\varepsilon^{\alpha}$-equilibria for some subnet $\left(X_{i}^{\alpha}, u_{i}^{\alpha}\right)$ and if $\left(u^{\alpha}\left(x^{\alpha}\right)\right)$ is a convergent net, then $x \in \mathcal{N}_{X}(u)$. This formulation of the result is useful for establishing the nonemptiness of $\mathcal{N}_{X}(u)$ since one need only find one net of $\varepsilon^{\alpha}$-equilibria for which $\left(x^{\alpha}, u^{\alpha}\left(x^{\alpha}\right)\right)$ is convergent. In applications, however, it is especially useful to know when the limit of any convergent sequence of approximate equilibria of approximating games is an equilibrium of the limit game. That is, it is useful to identify conditions that would ensure that $\varepsilon^{\nu} \rightarrow 0$ implies that

$$
\operatorname{Ls}\left(\mathcal{N}_{X^{\nu}}^{\varepsilon^{\nu}}\left(u^{\nu}\right)\right) \subseteq \mathcal{N}_{X}(u) .
$$

A result of this type that will be applied in our study of equilibrium refinements is the following.

Theorem 2. Suppose that $G=\left(X_{i}, u_{i}\right)$ is a topological game and suppose that $\left(X_{i}^{\nu}, u_{i}^{\nu}\right)$ is a test net for $G$ and suppose that $\left(u^{\nu}\right)$ is a uniformly bounded net in $U(X)$. The following are equivalent:

(1) G satisfies limit better-reply security with respect to $\left(X_{i}^{\nu}, u_{i}^{\nu}\right)$ (Condition 1).

(2) If $\varepsilon^{\nu} \rightarrow 0$, then $\operatorname{Ls}\left(\mathcal{N}_{X^{\nu}}^{\varepsilon^{\nu}}\left(u^{\nu}\right)\right) \subseteq \mathcal{N}_{X}(u)$.

Proof. Suppose that (1) is satisfied. Suppose that $\varepsilon^{\nu} \rightarrow 0$ and $x \in \operatorname{Ls}\left(\mathcal{N}_{X^{\nu}}^{\varepsilon^{\nu}}\left(u^{\nu}\right)\right)$. Then there exists a subnet $\left(\mathcal{N}_{X^{\alpha}}^{\varepsilon^{\alpha}}\left(u^{\alpha}\right)\right)$ and a net $\left(x^{\alpha}\right)$ satisfying $x^{\alpha} \in \mathcal{N}_{X^{\alpha}}^{\varepsilon^{\alpha}}\left(u^{\alpha}\right)$ for each $\alpha$ such that $x^{\alpha} \rightarrow x$. Since $\left(u^{\nu}\right)$ is a uniformly bounded net, there exists a further subnet $\left(x_{i}^{\beta}\right)$ of $\left(x_{i}^{\alpha}\right)$ such that $\left(x^{\beta}, u^{\beta}\left(x^{\beta}\right)\right)$ is a convergent net with limit $(x, \gamma) \in X \times \mathbb{R}^{N}$. Since $\varepsilon^{\nu} \rightarrow 0$ implies that $\varepsilon^{\beta} \rightarrow 0$ and since $\left(X_{i}^{\beta}, u_{i}^{\beta}\right)$ is a subnet of $\left(X_{i}^{\nu}, u_{i}^{\nu}\right)$, we can apply Theorem 1 and conclude that $x \in \mathcal{N}_{X}(u)$. This proves that (1) implies (2). The converse implication is a straightforward application of Theorem 1.

Remark 1. If $\left(u^{\nu}\right)$ is a convergent net in $U(X)$ with limit $u$, then $\left(u^{\nu}\right)$ is a uniformly bounded net in $U(X)$.

If $G=\left(X_{i}, u_{i}\right)$ is a topological game, then, letting $X^{\nu}=X$ and $u^{\nu}=u$ for all $\nu$, we obtain an immediate corollary of Theorem 2 .

Corollary 1. A topological game $G=\left(X_{i}, u_{i}\right)$ satisfies limit better-reply security if and only if $\varepsilon^{\nu} \rightarrow 0$ implies that $\operatorname{Ls}\left(\mathcal{N}_{X}^{\varepsilon^{\nu}}(u)\right) \subseteq \mathcal{N}_{X}(u)$. In particular, $\mathcal{N}_{X}(u)$ is a closed set if $G=\left(X_{i}, u_{i}\right)$ satisfies limit better-reply security. 


\section{Approximation results for mixed-strategy Nash equilibria of Borel games}

If $S$ is a topological space, let $\mathcal{B}(S)$ denote the class of Borel subsets of $S$. The cone of nonnegative, countably additive, regular measures on $\mathcal{B}(S)$ is denoted by $M_{+}(S)$. The subset of $M_{+}(S)$ consisting of probability measures endowed with the topology of weak convergence is denoted by $\Delta(S)$.

A topological game $G=\left(X_{i}, u_{i}\right)$ with each $u_{i}$ a bounded Borel measurable function is a Borel game. A topological game $G=\left(X_{i}, u_{i}\right)$ with each $X_{i}$ a separable (compact) metric space and each $u_{i}$ a bounded Borel measurable function is a separable (compact) metric Borel game.

The mixed extension of a Borel game $G$ is the strategic-form game

$$
\bar{G}:=\left(\Delta\left(X_{i}\right), u_{i}\right)_{i=1}^{N},
$$

where $u_{i}: \times_{i=1}^{N} \Delta\left(X_{i}\right) \rightarrow \mathbb{R}$ denotes the usual extension defined by

$$
u_{i}(\mu):=\int_{X} u_{i} d \mu_{1} \cdots d \mu_{n}
$$

We will abuse notation and define $\Delta(X):=\times_{i=1}^{N} \Delta\left(X_{i}\right)$. Next, define a correspondence $\mathcal{N}_{\Delta(X)}: U(X) \rightrightarrows \Delta(X)$ that assigns to each profile $u=$ $\left(u_{1}, . ., u_{N}\right) \in U(X)$ the set of Nash equilibria $\mathcal{N}_{\Delta(X)}(u)$ of the mixed extension $\bar{G}=\left(\Delta\left(X_{i}\right), u_{i}\right)$.

We now define analogues of Conditions 1 and 2 in terms of the mixed extension of a strategic-form game.

Condition 3. Suppose that $G=\left(X_{i}, u_{i}\right)$ is a Borel game with mixed extension $\bar{G}=\left(\Delta\left(X_{i}\right), u_{i}\right)$. Suppose that for each $i,\left(u_{i}^{\nu}\right)$ is a net of bounded, Borel measurable payoff functions and $\left(S_{i}^{\nu}\right)$ is a net of nonempty subsets of $\Delta\left(X_{i}\right)$. Then $\bar{G}$ satisfies limit better-reply security with respect to $\left(S_{i}^{\nu}, u_{i}^{\nu}\right)$ if the following condition is satisfied: if $\left(S_{i}^{\alpha}, u_{i}^{\alpha}\right)$ is a subnet of $\left(S_{i}^{\nu}, u_{i}^{\nu}\right)$, if $\left(\mu^{\alpha}, u^{\alpha}\left(\mu^{\alpha}\right)\right) \in \Delta(X) \times \mathbb{R}^{N}$ is a convergent net with limit $(\mu, \gamma) \in \Delta(X) \times \mathbb{R}^{N}$ satisfying $\mu^{\alpha} \in S^{\alpha}$ for each $\alpha$, and if $\mu$ is not a Nash equilibrium of $\bar{G}=\left(\Delta\left(X_{i}\right), u_{i}\right)$, then there exist $i, \eta>\gamma_{i}$, a subnet $\left(\mu^{\beta}\right)$ of $\left(\mu^{\alpha}\right)$ and a net $\left(p_{i}^{\beta}\right)$ such that for each $\beta, p_{i}^{\beta} \in S^{\beta}$ and $u_{i}\left(p_{i}^{\beta}, \mu_{-i}^{\beta}\right) \geq \eta$.

Condition 4 (limit better-reply security). Suppose that $G=\left(X_{i}, u_{i}\right)$ is a Borel game with mixed extension $\bar{G}=\left(\Delta\left(X_{i}\right), u_{i}\right)$. Then $\bar{G}$ satisfies limit 
better-reply security if the following condition is satisfied: if $\left(\mu^{\alpha}, u\left(\mu^{\alpha}\right)\right) \in$ $\Delta(X) \times \mathbb{R}^{N}$ is a convergent net with limit $(\mu, \gamma) \in \Delta(X) \times \mathbb{R}^{N}$ and if $\mu$ is not a Nash equilibrium of $\bar{G}=\left(\Delta\left(X_{i}\right), u_{i}\right)$, then there exist an $i$, an $\eta>\gamma_{i}$, a subnet $\left(\mu^{\beta}\right)$ of $\left(\mu^{\alpha}\right)$ and a net $\left(p_{i}^{\beta}\right)$ such that for each $\beta, p_{i}^{\beta} \in \Delta\left(X_{i}\right)$ and $u_{i}\left(p_{i}^{\beta}, \mu_{-i}^{\beta}\right) \geq \eta$.

The next result is an adaptation of Theorem 2 to the mixed extension of a game and the proof is the essentially identical.

Theorem 3. Suppose that $G=\left(X_{i}, u_{i}\right)$ is a Borel game. Suppose that for each $i,\left(u_{i}^{\nu}\right)$ is a uniformly bounded net of Borel measurable payoff functions and $\left(S_{i}^{\nu}\right)$ is a net of nonempty subsets of $\Delta\left(X_{i}\right)$. Suppose that $\bar{G}=$ $\left(\Delta\left(X_{i}\right), u_{i}\right)$ satisfies limit better-reply security with respect to $\left(S_{i}^{\nu}, u_{i}^{\nu}\right)$ (Condition 3). If $\left(S_{i}^{\alpha}, u_{i}^{\alpha}\right)$ is a subnet of $\left(S_{i}^{\nu}, u_{i}^{\nu}\right)$ and $\left(\mu^{\alpha}\right)$ is a convergent net in $\Delta(X)$ with limit $\mu \in \Delta(X)$ such that $\mu^{\alpha} \in \mathcal{N}_{S^{\alpha}}\left(u^{\alpha}\right)$ for each $\alpha$, then $\mu \in \mathcal{N}_{\Delta(X)}(u)$.

Corollary 2. Suppose that $\left(X_{i}, u_{i}\right)$ is a Borel game whose mixed extension satisfies limit better-reply security (Condition 4). Suppose that $\left(u^{\alpha}\right)$ is a uniformly bounded net of Borel measurable payoff functions and $\left(\mu^{\alpha}\right)$ is a convergent net in $\Delta(X)$ with limit $\mu \in \Delta(X)$ satisfying $\mu^{\alpha} \in \mathcal{N}_{\Delta(X)}\left(u^{\alpha}\right)$ for each $\alpha$. Then $\mu \in \mathcal{N}_{\Delta(X)}(u)$.

Proof. Apply Theorem 3 with $S_{i}^{\alpha}=\Delta\left(X_{i}\right)$ for each $\alpha$ and $i$.

We now state analogues of Conditions 3 and 4, Theorem 3, and Corollary 2 in terms of metric games.

Condition 5. Suppose that $G=\left(X_{i}, u_{i}\right)$ is a Borel, metric game with mixed extension $\bar{G}=\left(\Delta\left(X_{i}\right), u_{i}\right)$. Suppose that for each $i,\left(u_{i}^{n}\right)$ is a sequence of bounded, Borel measurable payoff functions and $\left(S_{i}^{n}\right)$ is a sequence of nonempty subsets of $\Delta\left(X_{i}\right)$. Then $\bar{G}$ satisfies sequential better-reply security with respect to $\left(S_{i}^{n}, u_{i}^{n}\right)$ if the following condition is satisfied: if $\left(S_{i}^{m}, u_{i}^{m}\right)$ is a subsequence of $\left(S_{i}^{n}, u_{i}^{n}\right)$, if $\left(\mu^{m}, u^{m}\left(\mu^{m}\right)\right) \in \Delta(X) \times \mathbb{R}^{N}$ is a convergent sequence with limit $(\mu, \gamma) \in \Delta(X) \times \mathbb{R}^{N}$ satisfying $\mu^{m} \in S^{m}$ for each $m$, and if $\mu$ is not a Nash equilibrium of $\bar{G}=\left(\Delta\left(X_{i}\right), u_{i}\right)$, then there exist $i, \eta>\gamma_{i}$, a subsequence $\left(\mu^{k}\right)$ of $\left(\mu^{m}\right)$ and a sequence $\left(p_{i}^{k}\right)$ such that for each $k, p_{i}^{k} \in S^{k}$ and $u_{i}\left(p_{i}^{k}, \mu_{-i}^{k}\right) \geq \eta$. 
Condition 6 (sequential better-reply security). Suppose that $G=$ $\left(X_{i}, u_{i}\right)$ is a Borel, metric game with mixed extension $\bar{G}=\left(\Delta\left(X_{i}\right), u_{i}\right)$. Then $\bar{G}$ satisfies sequential better-reply security if the following condition is satisfied: if $\left(\mu^{n}, u\left(\mu^{n}\right)\right) \in \Delta(X) \times \mathbb{R}^{N}$ is a convergent sequence with limit $(\mu, \gamma) \in \Delta(X) \times \mathbb{R}^{N}$ and if $\mu$ is not a Nash equilibrium of $\bar{G}=\left(\Delta\left(X_{i}\right), u_{i}\right)$, then there exist an $i$, an $\eta>\gamma_{i}$, a subsequence $\left(\mu^{n_{k}}\right)$ of $\left(\mu^{n}\right)$ and a sequence $\left(p_{i}^{k}\right)$ such that for each $k, p_{i}^{k} \in \Delta\left(X_{i}\right)$ and $u_{i}\left(p_{i}^{k}, \mu_{-i}^{n_{k}}\right) \geq \eta$.

Theorem 4. Suppose that $G=\left(X_{i}, u_{i}\right)$ is a Borel, metric game. Suppose that for each $i,\left(u_{i}^{n}\right)$ is a uniformly bounded sequence of Borel measurable payoff functions and $\left(S_{i}^{n}\right)$ is a sequence of nonempty subsets of $\Delta\left(X_{i}\right)$. Suppose that $\bar{G}$ satisfies sequential better-reply security with respect to $\left(S_{i}^{n}, u_{i}^{n}\right)$ (Condition 5). If $\left(S_{i}^{m}, u_{i}^{m}\right)$ is a subsequence of $\left(S_{i}^{n}, u_{i}^{n}\right)$ and $\left(\mu^{m}\right)$ is a convergent sequence in $\Delta(X)$ with limit $\mu \in \Delta(X)$ such that $\mu^{m} \in \mathcal{N}_{S^{m}}\left(u^{m}\right)$ for each $m$, then $\mu \in \mathcal{N}_{\Delta(X)}(u)$.

Corollary 3. Suppose that $\left(X_{i}, u_{i}\right)$ is a Borel metric game whose mixed extension satisfies sequential better-reply security (Condition 6). Suppose that $\left(u^{n}\right)$ is a uniformly bounded sequence of Borel measurable payoff functions and $\left(\mu^{n}\right)$ is a convergent net in $\Delta(X)$ with limit $\mu \in \Delta(X)$ satisfying $\mu^{n} \in \mathcal{N}_{\Delta(X)}\left(u^{n}\right)$ for each $n$. Then $\mu \in \mathcal{N}_{\Delta(X)}(u)$.

Proof. Apply Theorem 4 with $S_{i}^{n}=\Delta\left(X_{i}\right)$ for each $n$ and $i$.

\section{Application to perfect equilibrium}

Several authors have studied perfect equilibria in games with infinitely many actions (e.g., Simon and Stinchcombe [26], Al-Najjar [2], Carbonell-Nicolau $[9,10,11])$. The approximation results of Sections 3 and 4 can be used to derive new results on the existence of perfect equilibrium in discontinuous games.

Simon and Stinchcombe [26] present several extensions of Selten's [24] notion of perfection to games with infinitely many actions, including their concept of limit-of-finite (lof) perfect equilibrium. An lof perfect equilibrium is defined as the limit of $\epsilon$-perfect equilibria for successively finer finite approximations to an infinite game. It is shown in [26] that limit-of-finite perfection is ill-suited as a general solution concept even in continuous games. Before illustrating this idea, we introduce formal definitions of perfection, lof perfection, and admissibility. 
Throughout the sequel, unless otherwise indicated, we assume that $G=$ $\left(X_{i}, u_{i}\right)$ is a separable metric Borel game. In this case, the topology of weak convergence and the Prokhorov metric topology coincide and, consequently, sequences will be sufficient to define all weak limit concepts. In particular, $\Delta\left(X_{i}\right)$ is sequentially compact if $X_{i}$ is a compact metric space. If $X_{i}$ is only assumed to be metric, then $\Delta\left(X_{i}\right)$ is metrizable using the Prokhorov metric but the Prokhorov metric topology will be stronger than the topology of weak convergence and the latter may not be metrizable. Throughout the paper, we will abuse notation and use $\pi$ to denote the Prokhorov metric on both $\Delta\left(X_{i}\right)$ and the Cartesian product $\Delta(X)$.

A measure $\mu_{i} \in M_{+}\left(X_{i}\right)$ is strictly positive if $\mu_{i}(U)>0$ for every nonempty open set $U$ in $X_{i}$. Let $M_{++}\left(X_{i}\right)$ denote the set of all strictly positive measures in $M_{+}\left(X_{i}\right)$, let $\widehat{\Delta}\left(X_{i}\right)$ denote the set of all strictly positive probability measures in $\Delta\left(X_{i}\right)$, and let $\widehat{\Delta}(X):=\times_{i=1}^{N} \widehat{\Delta}\left(X_{i}\right)$. If $\eta_{i} \in M_{++}\left(X_{i}\right)$ and $0<\eta_{i}\left(X_{i}\right)<1$, we define the perturbed mixed strategy set of player $i$ as

$$
\Delta\left(X_{i}, \eta_{i}\right):=\left\{\nu_{i} \in \Delta\left(X_{i}\right): \nu_{i} \geq \eta_{i}\right\} .
$$

Given a profile $\eta=\left(\eta_{1}, \ldots, \eta_{N}\right) \in \times_{i=1}^{N} M_{++}\left(X_{i}\right)$ of perturbations, let $\Delta(X, \eta):=$ $\times_{i=1}^{N} \Delta\left(X_{i}, \eta_{i}\right)$. Define the associated Selten perturbation of $G$ to be the game

$$
\bar{G}_{\eta}=\left(\Delta\left(X_{i}, \eta_{i}\right), u_{i}\right)_{i=1}^{N} .
$$

Definition 2. Suppose that $G=\left(X_{i}, u_{i}\right)$ is a separable metric Borel game. A strategy profile $\mu \in \Delta(X)$ is trembling-hand perfect (thp) in $G$ if there exist a sequence of perturbation profiles $\left(\eta^{n}\right)$ and a sequence of mixed strategy profiles $\left(\mu^{n}\right)$ such that $\eta^{n} \rightarrow 0, \mu^{n} \rightarrow \mu$, and each $\mu^{n}$ is a Nash equilibrium of $\bar{G}_{\eta^{n}}$.

Thus, $\mu$ is a thp profile in $G$ if it is the limit of some sequence of Nash equilibria of neighboring Selten perturbations of $G$. It is important to note that a thp strategy profile for $G=\left(X_{i}, u_{i}\right)$ may not be a Nash equilibrium in the mixed extension $\bar{G}=\left(\Delta\left(X_{i}\right), u_{i}\right)$.

The reader is referred to Carbonell-Nicolau [10] for alternative, equivalent definitions of trembling-hand perfection.

Simon and Stinchcombe's [26] limit-of-finite perfect equilibrium is defined as follows. Let $G=\left(X_{i}, u_{i}\right)$ be a separable metric Borel game. For $Y_{i}$ a nonempty Borel subset of $X_{i}$ and for $\mu \in \Delta(X)$, let $B r_{i}\left(Y_{i}, \mu\right)$ denote player 
$i$ 's (possibly empty) set of best responses in $\Delta\left(Y_{i}\right)$ to the profile $\mu$ :

$$
B r_{i}\left(Y_{i}, \mu\right):=\left\{\sigma_{i} \in \Delta\left(Y_{i}\right): u_{i}\left(\sigma_{i}, \mu_{-i}\right)=\sup _{p_{i} \in \Delta\left(Y_{i}\right)} u_{i}\left(p_{i}, \mu_{-i}\right)\right\} .
$$

Definition 3 (Simon and Stinchcombe [26]). For each $i$ and $\delta>0$, let $X_{i}^{\delta}$ denote a finite subset of $X_{i}$ within Hausdorff distance $\delta$ of $X_{i}$. A profile $\mu^{(\epsilon, \delta)} \in \times_{i} \widehat{\Delta}\left(X_{i}^{\delta}\right)$ is $(\epsilon, \delta)$-perfect with respect to $\times_{i} X_{i}^{\delta}$ if for all $i$,

$$
d_{i}^{X_{i}^{\delta}}\left(\mu_{i}^{(\epsilon, \delta)}, B r_{i}\left(X_{i}^{\delta}, \mu^{(\epsilon, \delta)}\right)\right)<\epsilon,
$$

where

$$
d_{i}^{X_{i}^{\delta}}\left(\mu_{i}, \nu_{i}\right):=\sum_{x_{i} \in X_{i}^{\delta}}\left|\mu_{i}\left(\left\{x_{i}\right\}\right)-\nu_{i}\left(\left\{x_{i}\right\}\right)\right| .
$$

A strategy profile $\mu \in \Delta(X)$ in $G=\left(X_{i}, u_{i}\right)$ is limit-of-finite (lof) perfect in $G$ if it is the weak limit as $\left(\epsilon^{n}, \delta^{n}\right) \rightarrow 0$ of $\left(\epsilon^{n}, \delta^{n}\right)$-perfect profiles with respect to some sequence $\left(X^{\delta^{n}}\right)$.

Thus, $\mu$ is lof perfect strategy profile if it is the limit of $(\epsilon, \delta)$-perfect profiles for successively finer finite approximations of $G$.

Definition 4. A strategy $x_{i} \in X_{i}$ is weakly dominated for $i$ if there exists a strategy $\mu_{i} \in \Delta\left(X_{i}\right)$ such that $u_{i}\left(\mu_{i}, x_{-i}\right) \geq u_{i}\left(x_{i}, x_{-i}\right)$ for all $x_{-i} \in X_{-i}$, with strict inequality for some $x_{-i}$.

Definition 5. A strategy profile $\mu \in \Delta(X)$ is limit admissible if $\mu_{i}\left(W_{i}\right)=$ 0 for all $i$, where $W_{i}$ denotes the interior of the set of strategies weakly dominated for $i$.

Simon and Stinchcombe [26] provide an example of a continuous game in which each player has a single pure strategy that weakly dominates all other strategies. In this example, whether or not the dominant strategy is included in the finite approximations can drastically change the character of the game. For finite approximations that exclude this dominant strategy, a lof perfect profile may involve play of weakly dominated strategies, thereby violating limit admissibility. ${ }^{6}$

\footnotetext{
${ }^{6}$ This leads Simon and Stinchcombe to stregnthen the notion of lof perfection to anchored perfection. However, Simon and Stinchombe [26] claim that even anchored perfect profiles may fail limit admissibility in continuous games.
} 
Our limit-of-finite notion of perfection, which is a strengthening of Simon and Stinchcombe's [26] lof perfection, does satisfy limit admissibility within the class of continuous games (Subsection 5.2).

Definition 6. Let $G=\left(X_{i}, u_{i}\right)$ be a separable metric Borel game and let $\eta$ be a profile of perturbations. A finite $\varepsilon$-approximation of the Selten perturbation $\bar{G}_{\eta}$ of $G$ is a strategic-form game

$$
\bar{G}_{(Y, \xi)}^{\eta}=\left(\Delta\left(Y_{i}, \xi_{i}\right), u_{i}\right)
$$

where

- $Y_{i}$ is a finite subset of $X_{i}$ within Hausdorff distance $\varepsilon$ of $X_{i}$ for each $i$; and

- $\xi_{i}$ is a measure with finite support $Y_{i}$ such that $0<\xi_{i}\left(Y_{i}\right)<1$, $\left|\xi_{i}\left(Y_{i}\right)-\eta_{i}\left(X_{i}\right)\right|<\varepsilon$, and the Prokhorov distance between the probability measures $\frac{\xi_{i}}{\xi_{i}\left(Y_{i}\right)}$ and $\frac{\eta_{i}}{\eta_{i}\left(X_{i}\right)}$ is less than $\varepsilon$.

Remark 2. When $G=\left(X_{i}, u_{i}\right)$ is compact and metric, and given a Selten perturbation $\bar{G}_{\eta}$ of $G$, finite $\varepsilon$-approximations of $\bar{G}_{\eta}$ exist. To see this, note that $\operatorname{supp}\left(\frac{\eta_{i}}{\eta_{i}\left(X_{i}\right)}\right)=X_{i}$ since $\eta_{i} \in M_{++}\left(X_{i}\right)$. For each $i$, choose a sequence $\left(\sigma_{i}^{n}\right)$ of finitely supported measures in $\Delta\left(X_{i}\right)$ such that $\sigma_{i}^{n} \rightarrow \frac{\eta_{i}}{\eta_{i}\left(X_{i}\right)}$. By Lemma 2 below, there is a subsequence of $\left(\sigma_{i}^{n}\right)$, which we denote again by $\left(\sigma_{i}^{n}\right)$, such that $\left(\operatorname{supp}\left(\sigma_{i}^{n}\right)\right)$ converges to $X_{i}$ in the Hausdorff metric topology. Given $\varepsilon$, for large enough $n$,

$$
\left(\Delta\left(\operatorname{supp}\left(\sigma_{i}^{n}\right), \eta_{i}\left(X_{i}\right) \sigma_{i}^{n}\right), u_{i}\right)
$$

is a finite $\varepsilon$-approximation of $\bar{G}_{\eta}$.

Definition 7. Given sequences $\left(\varepsilon^{n}\right)$ and $\left(\eta^{n}\right)$, and a corresponding sequence $\left(\bar{G}_{\eta^{n}}\right)$ of Selten perturbations of $G$, the sequence $\left(\bar{G}_{\left(Y^{n}, \xi^{n}\right)}^{\eta^{n}}\right)$ is a finite $\left(\varepsilon^{n}\right)$ approximation of the sequence $\left(\bar{G}_{\eta^{n}}\right)$ if $\bar{G}_{\left(Y^{n}, \xi^{n}\right)}^{\eta^{n}}$ is an $\varepsilon^{n}$-approximation of $\bar{G}_{\eta^{n}}$ for each $n$.

Observe that the Selten perturbation $\bar{G}_{\eta}$, where $0<\eta_{i}\left(X_{i}\right)<1$ for each $i$, can be interpreted as a game in which each player $i$ is constrained to choose the mixed strategy $\frac{\eta_{i}}{\eta_{i}\left(X_{i}\right)}$ with probability $\eta_{i}\left(X_{i}\right)$, while the player is free to 
choose any mixed strategy in $\Delta\left(X_{i}\right)$ with probability $1-\eta_{i}\left(X_{i}\right)$. A similar interpretation applies to an approximation $\bar{G}_{(Y, \xi)}^{\eta}$ : each player $i$ can choose any mixed strategy in $\Delta\left(Y_{i}\right)$ with probability $1-\xi_{i}\left(Y_{i}\right)$ but is forced to play the mixed strategy $\frac{\xi_{i}}{\xi\left(Y_{i}\right)}$ with probability $\xi_{i}\left(Y_{i}\right)$.

Thus, when $\varepsilon$ is small, a finite $\varepsilon$-approximation $\bar{G}_{(Y, \xi)}^{\eta}$ of $\bar{G}_{\eta}$ is "close" to $\bar{G}_{\eta}$, in the sense that the mistakes the players make in $\bar{G}_{\eta}$ are "similar" to the mistakes they make in $\bar{G}_{(Y, \xi)}^{\eta}$, since the set of choices available in $\bar{G}_{(Y, \xi)}^{\eta}$ is "close" to the set of actions available in $\bar{G}_{\eta}$.

Definition 8. A strategy profile $\mu$ in $\Delta(X)$ is strongly limit-of-finite (lof) perfect in $G=\left(X_{i}, u_{i}\right)$ if there is a sequence $\left(\eta^{n}\right)$ with $\eta^{n} \rightarrow 0$ such that for every sequence $\left(\varepsilon^{n}\right)$ with $0<\varepsilon^{n} \rightarrow 0$, there exists a finite $\left(\varepsilon^{n}\right)$ approximation $\left(\bar{G}_{\left(Y^{n}, \xi^{n}\right)}^{\eta^{n}}\right)$ of $\left(\bar{G}_{\eta^{n}}\right)$ such that each $\bar{G}_{\left(Y^{n}, \xi^{n}\right)}^{\eta^{n}}$ possesses a Nash equilibrium $\mu^{n}$ and $\mu^{n} \rightarrow \mu$.

Thus, a strong lof perfect strategy profile is the limit of sequences of (exact) equilibria of neighboring finite Selten perturbations that respect the strategic aspects of the original (infinite) game, in the sense that they can be interpreted as "true" approximations of certain infinite Selten perturbations: If $\mu$ cannot be obtained as the limit of a sequence of equilibria extracted from some finite $\left(\varepsilon^{n}\right)$-approximation sufficiently close to some sequence $\left(\bar{G}_{\eta^{n}}\right)$ of (infinite) Selten perturbations of $G$, then the ability to approximate $\mu$ by a sequence of equilibria in finite "models of slight mistakes" critically relies on the sequence of finite perturbations being "far" from the infinite variants in the sequence $\left(\bar{G}_{\eta^{n}}\right)$ : either the trembles in the finite approximation are "far" from those in $\left(\bar{G}_{\eta^{n}}\right)$ or the approximation's finite action spaces place indispensable constraints on how the players can optimize their responses to the others' strategies and trembles (relative to their performance in $\left(\bar{G}_{\eta^{n}}\right)$ ). In either case, there is an essential "gap" between the sequence $\left(\bar{G}_{\eta^{n}}\right)$ and the finite $\left(\varepsilon^{n}\right)$-approximation used to approach $\mu$. We view this as an undesirable property of the approximating sequence because it is based on a fundamental inconsistency between the modeling of the original game and that of its Selten perturbations: the refinement specification is subject to "manipulation" via arbitrary omission (in the finite approximating sequence) of certain strategies otherwise available within the original game.

The following proposition establishes the relationship between strong lof perfection and Simon and Stinchcombe's [26] lof perfection. 
Proposition 1. If $G=\left(X_{i}, u_{i}\right)$ is a separable metric Borel game, then every strong limit-of-finite perfect profile is a limit-of-finite perfect profile.

Proof. Suppose that $\mu$ is a strong lof perfect profile in $G=\left(X_{i}, u_{i}\right)$. Then there exists a sequence $\left(\eta^{n}\right)$ with $\eta^{n} \rightarrow 0$ such that, for every sequence $\left(\varepsilon^{n}\right)$ with $0<\varepsilon^{n} \rightarrow 0$, there exists a finite $\left(\varepsilon^{n}\right)$-approximation $\left(\bar{G}_{\left(Y^{n}, \xi^{n}\right)}^{\eta^{n}}\right)$ of $\left(\bar{G}_{\eta^{n}}\right)$ such that each $\bar{G}_{\left(Y^{n}, \xi^{n}\right)}^{\eta^{n}}$ possesses a Nash equilibrium $\mu^{n}$ with $\mu^{n} \rightarrow \mu$. We claim that $\mu$ is a lof perfect strategy profile. Observe that each $\left(Y_{i}^{n}\right)$ (Hausdorff) converges to $X_{i}$, and that $\eta_{i}^{n} \rightarrow 0$ for each $i$. To complete the proof, we must show that for each $i$ the distance (as defined in Definition 3) between

$$
\mu_{i}^{n} \in \underset{p \in \Delta\left(Y_{i}^{n}, \xi_{i}^{n}\right)}{\arg \max } u_{i}\left(p, \mu_{-i}^{n}\right)
$$

and

$$
\operatorname{Br}_{i}\left(Y_{i}^{n}, \mu^{n}\right)=\underset{p \in \Delta\left(Y_{i}^{n}\right)}{\arg \max } u_{i}\left(p, \mu_{-i}^{n}\right)
$$

converges to 0 . This will be true if each $\mu_{i}^{n}$ can be expressed as

$$
\mu_{i}^{n}:=\left(1-\delta^{n}\right) \rho_{i}^{n}+\delta^{n} p_{i}^{n},
$$

for some sequence $\left(\delta^{n}\right)$ with $[0,1] \ni \delta^{n} \rightarrow 0$, some $\rho_{i}^{n} \in B r_{i}\left(Y_{i}^{n}, \mu^{n}\right)$, and some $p_{i}^{n} \in \Delta\left(Y_{i}^{n}\right)$, since, in that case,

$$
\begin{aligned}
d_{i}^{Y_{i}^{n}}\left(\mu_{i}^{n}, \rho_{i}^{n}\right) & =\sum_{x_{i} \in Y_{i}^{n}}\left|\mu_{i}^{n}\left(\left\{x_{i}\right\}\right)-\rho_{i}^{n}\left(\left\{x_{i}\right\}\right)\right| \\
& =\delta^{n} \sum_{x_{i} \in Y_{i}^{n}}\left|\rho_{i}^{n}\left(\left\{x_{i}\right\}\right)-p_{i}^{n}\left(\left\{x_{i}\right\}\right)\right| \\
& \leq 2 \delta^{n} .
\end{aligned}
$$

To see this, write $\mu_{i}^{n}=\hat{\mu}_{i}^{n}+\xi_{i}^{n}$, where $\hat{\mu}_{i}^{n}:=\mu_{i}^{n}-\xi_{i}^{n}$. Since $1>\xi_{i}^{n}\left(Y_{i}^{n}\right)>$ $0, \hat{\mu}_{i}^{n}\left(Y_{i}^{n}\right)=\mu_{i}^{n}\left(Y_{i}^{n}\right)-\xi_{i}^{n}\left(Y_{i}^{n}\right)=1-\xi_{i}^{n}\left(Y_{i}^{n}\right)>0$, and we have

$$
\mu_{i}^{n}=\hat{\mu}_{i}^{n}\left(Y_{i}^{n}\right) \frac{\hat{\mu}_{i}^{n}}{\hat{\mu}_{i}^{n}\left(Y_{i}^{n}\right)}+\xi_{i}^{n}\left(Y_{i}^{n}\right) \frac{\xi_{i}^{n}}{\xi_{i}^{n}\left(Y_{i}^{n}\right)}=\left(1-\xi_{i}^{n}\left(Y_{i}^{n}\right)\right) \frac{\hat{\mu}_{i}^{n}}{\hat{\mu}_{i}^{n}\left(Y_{i}^{n}\right)}+\xi_{i}^{n}\left(Y_{i}^{n}\right) \frac{\xi_{i}^{n}}{\xi_{i}^{n}\left(Y_{i}^{n}\right)} .
$$

Since $\hat{\mu}_{i}^{n}\left(Y_{i}^{n}\right)+\xi_{i}^{n}\left(Y_{i}^{n}\right)=\mu_{i}^{n}\left(Y_{i}^{n}\right)=1$ and $\xi_{i}^{n}\left(Y_{i}^{n}\right) \rightarrow 0$, it only remains to show that $\frac{\hat{\mu}_{i}^{n}}{\hat{\mu}_{i}^{n}\left(Y_{i}^{n}\right)} \in B r_{i}\left(Y_{i}^{n}, \mu^{n}\right)$. If $\frac{\hat{\mu}_{i}^{n}}{\hat{\mu}_{i}^{n}\left(Y_{i}^{n}\right)} \notin B r_{i}\left(Y_{i}^{n}, \mu^{n}\right)$, then there exists a $\rho_{i} \in B_{i}\left(Y_{i}^{n}, \mu^{n}\right)$ such that

$$
u_{i}\left(\rho_{i}, \mu_{-i}^{n}\right)>u_{i}\left(\frac{\hat{\mu}_{i}^{n}}{\hat{\mu}_{i}^{n}\left(Y_{i}^{n}\right)}, \mu_{-i}^{n}\right) .
$$


Using (1) together with linearity, we can rearrange this inequality to obtain

$$
u_{i}\left(\hat{\mu}_{i}^{n}\left(Y_{i}^{n}\right) \rho_{i}+\xi_{i}^{n}\left(Y_{i}^{n}\right) \frac{\xi_{i}^{n}}{\xi_{i}^{n}\left(Y_{i}^{n}\right)}, \mu_{-i}^{n}\right)>u_{i}\left(\mu_{i}^{n}, \mu_{-i}^{n}\right),
$$

contradicting the assumption that

$$
\mu_{i}^{n} \in \underset{p \in \Delta\left(Y_{i}^{n}, \xi_{i}^{n}\right)}{\arg \max } u_{i}\left(p, \mu_{-i}^{n}\right)
$$

This completes the proof.

Remark 3. Simon and Stinchcombe [26] define lof perfection in terms of $\epsilon$-perfect equilibria, while strong lof perfection is defined in terms of finite approximations of Selten perturbations. There is, however, an alternative formulation of lof perfection in terms of finite Selten perturbations. In fact, it can be shown that Definition 3 is equivalent to the following: A strategy profile $\mu \in \Delta(X)$ is lof perfect in $G=\left(X_{i}, u_{i}\right)$ if there are sequences $\left(X^{n}\right)=$ $\left(X_{1}^{n}, \ldots, X_{N}^{n}\right),\left(\xi^{n}\right)$, and $\left(\mu^{n}\right)$ such that each $\xi_{i}^{n}$ has support $X_{i}^{n}, \xi^{n} \rightarrow 0$, each $X_{i}^{n} \subseteq X_{i}$ is finite, $X_{i}^{n} \rightarrow X_{i}$ for each $n$ and $i, \mu^{n} \rightarrow \mu$, and each $\mu^{n}$ is a Nash equilibrium of $\bar{G}_{\left(Y^{n}, \xi^{n}\right)}^{\eta^{n}}$.

This alternative formulation of lof perfection is more easily compared with strong lof perfection (Definition 8).

An lof perfect strategy profile in $G=\left(X_{i}, u_{i}\right)$ need not be a mixedstrategy equilibrium, even if an lof perfect strategy profile exists for $G=$ $\left(X_{i}, u_{i}\right)$. We first address the question of existence of strong lof perfect equilibrium profiles. In Subsection 5.2 we discuss some properties of strong lof perfection.

\subsection{Existence of strong limit-of-finite perfect equilib- rium}

Proposition 2 below establishes the relationship between the approximation results of Section 4 and the existence of strong lof perfect equilibria. Proposition 2 allows us to prove Theorems 5 and 6 , which are existence results formulated in terms of the data of the original game, $G=\left(X_{i}, u_{i}\right)$.

The proof of Proposition 2 relies on two auxiliary results. Before stating these results, we introduce some terminology. 
Given $(\delta, \mu) \in[0,1)^{N} \times \Delta(X)$, let

$$
G_{(\delta, \mu)}:=\left(X_{i}, u_{i}^{(\delta, \mu)}\right)_{i=1}^{N}
$$

be the strategic-form game where the payoff function $u_{i}^{(\delta, \mu)}: X \rightarrow \mathbb{R}$ is defined by

$$
u_{i}^{(\delta, \mu)}(x):=u_{i}\left(\left(1-\delta_{1}\right) x_{1}+\delta_{1} \mu_{1}, \ldots,\left(1-\delta_{N}\right) x_{N}+\delta_{N} \mu_{N}\right) .
$$

Here, $\left(1-\delta_{i}\right) x_{i}+\delta_{i} \mu_{i}$ denotes the measure $\sigma_{i} \in \Delta\left(X_{i}\right)$ defined by $\sigma_{i}(B):=$ $\left(1-\delta_{i}\right) \chi_{x_{i}}(B)+\delta_{i} \mu_{i}(B)$ where $\chi_{x_{i}} \in \Delta\left(X_{i}\right)$ is the Dirac measure with support $\left\{x_{i}\right\}$.

Given $(\nu, \mu) \in \Delta(X) \times \Delta(X)$, the vector

$$
\left(\left(1-\delta_{1}\right) \nu_{1}+\delta_{1} \mu_{1}, \ldots,\left(1-\delta_{N}\right) \nu_{N}+\delta_{N} \mu_{N}\right)
$$

is sometimes denoted as $(1-\delta) \nu+\delta \mu$.

Lemma 1. Suppose that $\left(X_{i}, u_{i}\right)$ is a separable, metric, Borel game. Suppose that $Q_{i} \subseteq X_{i}$ is countable for each $i$. If $(\delta, \mu) \in[0,1)^{N} \times \Delta(X)$ and $[0,1)^{N} \ni$ $\delta^{n} \rightarrow \delta$, then there exists a sequence $\left(\mu^{n}\right)$ with $\Delta(X) \ni \mu^{n} \rightarrow \mu$ such that each $\mu_{i}^{n}$ has finite support $X_{i}^{n}, X_{i}^{n} \subseteq X_{i}^{n+1}$ for each $n$ and $i$, and $\bigcup_{n=1}^{\infty} X_{i}^{n} \supseteq Q_{i}$ for each $i$.

Proof. For each $i$, let $\left(\nu_{i}^{n}\right)$ be sequence with $\Delta\left(X_{i}\right) \ni \nu_{i}^{n} \rightarrow \mu_{i}$ such that each $\nu_{i}^{n}$ has finite support $Y_{i}^{n}$. Let $Q_{i}=\left\{q_{i}^{n}\right\}_{n=1}^{\infty}$. Next define

$$
X_{i}^{n}=\left[\cup_{k=1}^{n} Y_{i}^{n}\right] \cup\left\{q_{i}^{1}, . ., q_{i}^{n}\right\}
$$

and note that each $X_{i}^{n}$ is finite, $X_{i}^{n} \subseteq X_{i}^{n+1}$ and $\bigcup_{n=1}^{\infty} X_{i}^{n} \supseteq Q_{i}$. For each $i$ and $n$, define $\mu_{i}^{n} \in \Delta\left(X_{i}\right)$ as follows:

$$
\mu_{i}^{n}:=\left(\frac{n-1}{n}\right) \nu_{i}^{n}+\frac{1}{n^{2}} \sum_{k=1}^{n}\left(\frac{\nu_{i}^{k}+\chi_{\left\{q_{i}^{k}\right\}}}{2}\right) .
$$

Note that each $\mu_{i}^{n}$ has support $X_{i}^{n}$. To show that $\mu_{i}^{n} \rightarrow \mu_{i}$, suppose that $f: X_{i} \rightarrow \mathbb{R}$ is continuous and bounded with bound $M$. Then

$$
\left|\int_{X_{i}} f d \mu_{i}^{n}-\int_{X_{i}} f d \mu_{i}\right| \leq\left|\int_{X_{i}} f d \nu_{i}^{n}-\int_{X_{i}} f d \mu_{i}\right|+\frac{M}{n}+\frac{n M}{n^{2}} \rightarrow 0 \text { as } n \rightarrow \infty .
$$


Since each $X_{i}$ is a separable metric space, we can apply Theorem 3.2 in Billingsley [8] and conclude that

$$
\mu^{n}=\mu_{1}^{n} \otimes \cdots \otimes \mu_{N}^{n} \rightarrow \mu_{1} \otimes \cdots \otimes \mu_{N}=\mu,
$$

as desired.

Lemma 2. Let $X$ be a compact metric space and suppose that $\left(\mu^{n}\right)$ is a sequence in $\Delta(X)$ weakly converging to $\mu \in \Delta(X)$. Then there exists a subsequence which we also denote by $\left(\mu^{n}\right)$ and a set $S \subseteq X$ such that $\operatorname{supp}(\mu) \subseteq S$ and $\left(\operatorname{supp}\left(\mu^{n}\right)\right)$ is convergent in the Hausdorff metric topology with limit $S$.

Proof. Since $X$ is a compact metric space, the hyperspace of nonempty compact subsets of $X$ is a compact metric space with respect to the Hausdorff metric. The set $\operatorname{supp}\left(\mu^{n}\right)$ is closed, hence compact in $X$. Consequently, there exists a subsequence which we also denote by $\left(\mu^{n}\right)$ and a compact set $S \subseteq X$ such that $\operatorname{supp}\left(\mu^{n}\right) \rightarrow S$. To see that $\operatorname{supp}(\mu) \subseteq S$, suppose that there exists $x \in \operatorname{supp}(\mu) \backslash S$. Then since $S$ is closed there exist a neighborhood $V_{x}$ of $x$ and an open set $U$ containing $S$ such that $V_{x} \cap U=\emptyset$. Note that $\mu\left(V_{x}\right)>0$ since $x \in \operatorname{supp}(\mu)$. Since $V_{x} \cap U=\emptyset$ and $\operatorname{supp}\left(\mu^{n}\right) \rightarrow S$ it follows that $\mu^{n}\left(V_{x}\right)=0$ for any large enough $n$. On the other hand, since $\mu^{n} \rightarrow \mu$ and $\mu\left(V_{x}\right)>0$, we have $0=\underline{\lim } \mu^{n}\left(V_{x}\right) \geq \mu\left(V_{x}\right)$, an impossibility.

Proposition 2. Suppose that $G=\left(X_{i}, u_{i}\right)$ is a compact, metric, Borel game. Suppose further that

(i) $\bar{G}$ satisfies sequential better-reply security (Condition 6), and

(ii) there exist sequences $\left(\nu^{n}\right)$ and $\left(\delta^{n}\right)$ with $\nu^{n} \in \widehat{\Delta}(X)$ and $(0,1)^{N} \ni$ $\delta^{n} \rightarrow 0$ and, for each $i$, a countable subset $Q_{i}$ of $X_{i}$ such that for each $n$, the following condition holds: $\left(\Delta\left(X_{i}\right), u_{i}^{\left(\delta^{n}, \nu^{n}\right)}\right)$ satisfies sequential better-reply security with respect to any sequence

$$
\left(\Delta\left(X_{i}^{n}(m)\right), u_{i}^{\left(\delta^{n}, \sigma^{n}(m)\right)}\right)_{m \geq 1}
$$

(Condition 5), where $\sigma^{n}(m)=\left(\sigma_{1}^{n}(m), \ldots \sigma_{N}^{n}(m)\right) \in \Delta(X)$ and for each $i,\left(X_{i}^{n}(m)\right)_{m \geq 1}$ is an increasing sequence of finite subsets of $X_{i}, X_{i}^{n}(m)$ is the finite support of $\sigma_{i}^{n}(m)$,

$$
\begin{array}{r}
\bigcup_{m=1}^{\infty} X_{i}^{n}(m) \supseteq Q_{i}, \\
X_{i}^{n}(m) \underset{m \rightarrow \infty}{\longrightarrow} X_{i}, \text { and } \sigma^{n}(m) \underset{m \rightarrow \infty}{\longrightarrow} \nu^{n} .
\end{array}
$$


Then $G$ possesses a strong limit-of-finite perfect equilibrium, which is also trembling-hand perfect.

Proof. Suppose that $\left(X_{i}, u_{i}\right)$ is a compact, metric, Borel game. Assume $(i)$ and $(i i)$ above.

For each $n$, we can apply Lemma 1 and deduce the existence of a sequence of games

$$
\left(\Delta\left(X_{i}^{n}(m)\right), u_{i}^{\left(\delta^{n}, \sigma^{n}(m)\right)}\right)_{m \geq 1}
$$

with the following properties:

- for each $i, X_{i}^{n}(m) \subseteq X_{i}$ is the finite support of $\sigma_{i}^{n}(m)$;

- for each $i, X_{i}^{n}(m) \subseteq X_{i}^{n}(m+1)$ for each $m$;

- for each $i, \bigcup_{m=1}^{\infty} X_{i}^{n}(m) \supseteq Q_{i}$; and

- $\sigma^{n}(m) \underset{m \rightarrow \infty}{\longrightarrow} \nu^{n}$.

Since $\nu^{n} \in \widehat{\Delta}(X)$ implies that $\operatorname{supp}\left(\nu_{i}^{n}\right)=X_{i}$ for each $i$, we can apply Lemma 2 and assume (passing to a subsequence if necessary) that $X_{i}^{n}(m) \underset{m \rightarrow \infty}{\longrightarrow}$ $X_{i}$.

Applying (ii), it follows that the game $\bar{G}=\left(\Delta\left(X_{i}\right), u_{i}^{\left(\delta^{n}, \nu^{n}\right)}\right)$ satisfies sequential better-reply security (i.e., Condition 5 ) with respect to the sequence in $(2)$.

For each $m$, the game $\left(\Delta\left(X_{i}^{n}(m)\right), u_{i}^{\left(\delta^{n}, \sigma^{n}(m)\right)}\right)$ has a Nash equilibrium $\mu^{n}(m)$, and (because $\Delta(X)$ is sequentially compact) we have (passing to a subsequence if necessary) $\mu^{n}(m) \underset{m \rightarrow \infty}{\longrightarrow} \mu^{n}$ for some $\mu^{n} \in \Delta(X)$. Since $\left(u^{\left(\delta^{n}, \sigma^{n}(m)\right)}\right)_{m=1}^{\infty}$ is a uniformly bounded sequence, Theorem 4 implies that $\mu^{n} \in \mathcal{N}_{\Delta(X)}\left(u^{\left(\delta^{n}, \nu^{n}\right)}\right)$. Since $\Delta\left(X_{i}\right)$ is sequentially compact, we conclude (extracting a subsequence if necessary) that there exists a $\mu \in \Delta(X)$ such that $\mu^{n} \rightarrow \mu$. To show that $\mu$ is a thp equilibrium, first observe that the sequence $\left(u^{\left(\delta^{n}, \nu^{n}\right)}\right)$ is uniformly bounded. Applying Corollary 3, it follows that $\mu \in \mathcal{N}_{\Delta(X)}(u)$. Next, define $q_{i}^{n}:=\left(1-\delta_{i}^{n}\right) \mu_{i}^{n}+\delta_{i}^{n} \nu_{i}^{n}$ for each $i$ and note that $q^{n}=\left(q_{1}^{n}, . ., q_{N}^{n}\right)$ is a Nash equilibrium in the Selten perturbation $\left(\Delta\left(X_{i}, \delta_{i}^{n} \nu_{i}^{n}\right), u_{i}\right)$. Since $q^{n} \rightarrow \mu$, we conclude that $\mu$ is a thp profile and a Nash equilibrium.

Since $\mu$ is a Nash equilibrium, the proof will be complete if we show that $\mu$ is a strong lof strategy profile. To begin, note that $\left(\Delta\left(X_{i}, \delta_{i}^{n} \nu_{i}^{n}\right), u_{i}\right)_{i=1}^{N}$ is a 
Selten perturbation of $G$ and that $\delta_{i}^{n} \nu_{i}^{n} \rightarrow 0$ for each $i$. Let $\left(\varepsilon^{n}\right)$ be a sequence of positive numbers satisfying $\varepsilon^{n} \rightarrow 0$. To show that $\mu$ is a strong lof strategy profile, we first construct a finite $\left(\varepsilon^{n}\right)$-approximation of $\left(\left(\Delta\left(X_{i}, \delta_{i}^{n} \nu_{i}^{n}\right), u_{i}\right)_{i=1}^{N}\right)$.

Defining

$$
p_{i}^{n}(m):=\left(1-\delta_{i}^{n}\right) \mu_{i}^{n}(m)+\delta_{i}^{n} \sigma_{i}^{n}(m),
$$

it follows that $p^{n}(m):=\left(p_{1}^{n}(m), . ., p_{N}^{n}(m)\right)$ is a Nash equilibrium of the (finite) Selten perturbation $\left(\Delta\left(X_{i}^{n}(m), \delta_{i}^{n} \sigma_{i}^{n}(m)\right), u_{i}\right)$. For each $n$, choose $m_{n}^{*}$ so that for each $i$, haus $_{i}\left(X_{i}^{n}\left(m_{n}^{*}\right), X_{i}\right)<\varepsilon^{n}, \pi\left(\sigma_{i}^{n}\left(m_{n}^{*}\right), \nu_{i}^{n}\right)<\varepsilon^{n}$ and $\pi\left(\mu_{i}^{n}\left(m_{n}^{*}\right), \mu_{i}^{n}\right)<$ $\varepsilon^{n}$. Here, $\operatorname{haus}_{i}(\cdot, \cdot)$ denotes the Hausdorff distance induced by the metric on $X_{i}$. Since haus $\left(X_{i}^{n}\left(m_{n}^{*}\right), X_{i}\right)<\varepsilon^{n}$ and $\pi\left(\sigma_{i}^{n}\left(m_{n}^{*}\right), \nu_{i}^{n}\right)<\varepsilon^{n}$, it follows that the sequence $\left(\Delta\left(X_{i}^{n}\left(m_{n}^{*}\right), \delta_{i}^{n} \sigma_{i}^{n}\left(m_{n}^{*}\right)\right), u_{i}\right)_{n \geq 1}$ is a finite $\left(\varepsilon^{n}\right)$-approximation of $\left(\left(\Delta\left(X_{i}, \delta_{i}^{n} \nu_{i}^{n}\right), u_{i}\right)_{i=1}^{N}\right)$. Furthermore, $p^{n}\left(m_{n}^{*}\right) \in \Delta\left(X_{i}^{n}\left(m_{n}^{*}\right)\right)$ defined for each $i$ as $p_{i}^{n}\left(m_{n}^{*}\right)=\left(1-\delta_{i}^{n}\right) \mu_{i}^{n}\left(m_{n}^{*}\right)+\delta_{i}^{n} \sigma^{n}\left(m_{n}^{*}\right)$ is an equilibrium of the (finite) Selten perturbed game

$$
\left(\Delta\left(X_{i}^{n}\left(m_{n}^{*}\right), \delta_{i}^{n} \sigma_{i}^{n}\left(m_{n}^{*}\right)\right), u_{i}\right)
$$

Since $\pi\left(\mu_{i}^{n}\left(m_{n}^{*}\right), \mu_{i}^{n}\right)<\varepsilon^{n}, \delta^{n} \rightarrow 0$ and $\mu^{n} \rightarrow \mu$, we conclude that $p^{n}\left(m_{n}^{*}\right) \rightarrow \mu$ implying that $\mu$ is a strong limit-of-finite strategy profile.

We now seek conditions on the payoff functions of the original game $\left(X_{i}, u_{i}\right)$, rather than perturbations of the form $G_{(\delta, \mu)}$, that imply the hypothesis of Proposition 2.

The following condition is taken from Carbonell-Nicolau [11].

Condition (A). There exists $\left(\nu_{1}, \ldots, \nu_{N}\right) \in \widehat{\Delta}(X)$ such that for each $i$ and every $\varepsilon>0$ there is a Borel measurable map $f_{i}^{\varepsilon}: X_{i} \rightarrow X_{i}$ such that the following is satisfied:

(a) For each $x_{i} \in X_{i}$ and every $y_{-i} \in X_{-i}$, there is a neighborhood $O_{y_{-i}}$ of $y_{-i}$ such that $u_{i}\left(f_{i}^{\varepsilon}\left(x_{i}\right), z_{-i}\right)>u_{i}\left(x_{i}, y_{-i}\right)-\varepsilon$ for every $z_{-i} \in O_{y_{-i}}$.

(b) For each $y_{-i} \in X_{-i}$, there is a subset $Y_{i}$ of $X_{i}$ with $\nu_{i}\left(Y_{i}\right)=1$ such that for every $x_{i} \in Y_{i}$, there is a neighborhood $V_{y_{-i}}$ of $y_{-i}$ such that $u_{i}\left(f_{i}^{\varepsilon}\left(x_{i}\right), z_{-i}\right)<u_{i}\left(x_{i}, z_{-i}\right)+\varepsilon$ for all $z_{-i} \in V_{y_{-i}}$.

In this paper we need the following strengthening of Condition (A). 
Condition $\left(\mathbf{A}^{*}\right)$. There exists $\left(\nu_{1}, \ldots, \nu_{N}\right) \in \widehat{\Delta}(X)$ such that for each $i$ and every $\varepsilon>0$ there is a Borel measurable map $f_{i}^{\varepsilon}: X_{i} \rightarrow X_{i}$ with countable range such that the following is satisfied:

(a) For each $x_{i} \in X_{i}$ and every $y_{-i} \in X_{-i}$, there is a neighborhood $O_{y_{-i}}$ of $y_{-i}$ such that $u_{i}\left(f_{i}^{\varepsilon}\left(x_{i}\right), z_{-i}\right)>u_{i}\left(x_{i}, y_{-i}\right)-\varepsilon$ for every $z_{-i} \in O_{y_{-i}}$.

(b) For each $y_{-i} \in X_{-i}$, there is a subset $Y_{i}$ of $X_{i}$ with $\nu_{i}\left(Y_{i}\right)=1$ such that for every $x_{i} \in Y_{i}$, there is a neighborhood $V_{y_{-i}}$ of $y_{-i}$ such that $u_{i}\left(f_{i}^{\varepsilon}\left(x_{i}\right), z_{-i}\right)<u_{i}\left(x_{i}, z_{-i}\right)+\varepsilon$ for all $z_{-i} \in V_{y_{-i}}$.

Condition $\left(\mathrm{A}^{*}\right)$ is Condition $(\mathrm{A})$ with the added constraint that the range of the map $f_{i}^{\varepsilon}$ be countable. Condition $\left(\mathrm{A}^{*}\right)$ is used to prove Theorem 5 , and the proof of this result makes it clear where the countability requirement is used. Theorem 5 states that compact games satisfying Condition $\left(\mathrm{A}^{*}\right)$ and upper semicontinuity of the sum of payoffs possess strong lof perfect equilibria. Theorem 6 (below) is formulated in terms of two easily-verified conditions, generic entire payoff security and generic local equi-upper semicontinuity, introduced in Carbonell-Nicolau [11]. These conditions prove useful in many applications (see Remark 5 below).

Lemma 3. Suppose that $G=\left(X_{i}, u_{i}\right)$ is a compact, metric, Borel game satisfying Condition $\left(\mathrm{A}^{*}\right)$. Then, there exist $\nu \in \widehat{\Delta}(X)$, and, for each $i$, a countable set $Q_{i} \subseteq X_{i}$ such that the following is satisfied: given $\delta \in[0,1)$, $n \in \mathbb{N}$, $i$, and $\sigma=\left(\sigma_{1}, \ldots, \sigma_{N}\right) \in \Delta(X, \delta \nu)$, there exists $\varrho_{\text {in }} \in \Delta\left(X_{i}\right)$ with $\varrho_{i n}\left(Q_{i}\right)=1$ and a neighborhood $O_{\sigma_{-i}}$ of $\sigma_{-i}$ such that

$$
u_{i}\left((1-\delta) \varrho_{i n}+\delta \nu_{i}, p_{-i}\right)>u_{i}(\sigma)-\frac{1}{n}, \quad \text { for all } p_{-i} \in O_{\sigma_{-i}} .
$$

Proof. Suppose that $G=\left(X_{i}, u_{i}\right)$ is a compact, metric, Borel game satisfying Condition $\left(\mathrm{A}^{*}\right)$. By Condition $\left(\mathrm{A}^{*}\right)$, there exists $\nu \in \widehat{\Delta}(X)$ such that for each $i$ and every $n \in \mathbb{N}$ there is a Borel measurable map $f_{i n}: X_{i} \rightarrow X_{i}$ with countable range such that the following is satisfied:

(a) For each $x_{i} \in X_{i}$ and every $y_{-i} \in X_{-i}$, there is a neighborhood $O_{y_{-i}}$ of $y_{-i}$ such that $u_{i}\left(f_{i n}\left(x_{i}\right), z_{-i}\right)>u_{i}\left(x_{i}, y_{-i}\right)-\frac{1}{n}$ for every $z_{-i} \in O_{y_{-i}}$.

(b) For each $y_{-i} \in X_{-i}$, there is a subset $Y_{i}$ of $X_{i}$ with $\nu_{i}\left(Y_{i}\right)=1$ such that for every $x_{i} \in Y_{i}$, there is a neighborhood $V_{y_{-i}}$ of $y_{-i}$ such that $u_{i}\left(f_{i n}\left(x_{i}\right), z_{-i}\right)<u_{i}\left(x_{i}, z_{-i}\right)+\frac{1}{n}$ for all $z_{-i} \in V_{y_{-i}}$. 
For each $i$, define

$$
Q_{i}:=\bigcup_{n=1}^{\infty} f_{i n}\left(X_{i}\right) .
$$

Since each $f_{i n}$ has a countable range, $Q_{i}$ is countable. Consequently, the proof will be complete if we show that given $\delta \in[0,1), n \in \mathbb{N}$, $i$, and $\sigma=\left(\sigma_{1}, \ldots, \sigma_{N}\right) \in \Delta(X, \delta \nu)$, there exists $\varrho_{i n} \in \Delta\left(X_{i}\right)$ with $\varrho_{i n}\left(Q_{i}\right)=1$ and a neighborhood $O_{\sigma_{-i}}$ of $\sigma_{-i}$ such that (3) holds. Fix $\delta \in[0,1), n \in \mathbb{N}, i$, and $\sigma=\left(\sigma_{1}, \ldots, \sigma_{N}\right) \in \Delta(X, \delta \nu)$. From the proof of Lemma 2 in CarbonellNicolau [9], it follows that there exist $\varrho_{\text {in }} \in \Delta\left(X_{i}\right)$ with $\varrho_{i n}\left(f_{i n}\left(X_{i}\right)\right)=1$ and a neighborhood $O_{\sigma_{-i}}$ of $\sigma_{-i}$ such that

$$
u_{i}\left((1-\delta) \varrho_{i n}+\delta \nu_{i}, p_{-i}\right)>u_{i}(\sigma)-\frac{1}{n}, \quad \text { for all } p_{-i} \in O_{\sigma_{-i}} .
$$

Since $1=\varrho_{i n}\left(f_{i n}\left(X_{i}\right)\right) \leq \varrho_{i n}\left(Q_{i}\right) \leq 1$, the proof is complete.

Theorem 5. Suppose that $G=\left(X_{i}, u_{i}\right)$ is a compact, metric, Borel game satisfying Condition ( $\left.\mathrm{A}^{*}\right)$. Suppose further that $\sum_{i} u_{i}$ is upper semicontinuous. Then $G$ possesses a strong limit-of-finite perfect equilibrium, which is also trembling-hand perfect.

Proof. Suppose that $G=\left(X_{i}, u_{i}\right)$ is a compact, metric, Borel game satisfying Condition $\left(\mathrm{A}^{*}\right)$. Suppose further that $\sum_{i} u_{i}$ is upper semicontinuous. It suffices to show that the hypotheses of Proposition 2 are satisfied.

That $\bar{G}$ satisfies Condition 6 (in fact better-reply security) follows from Condition $\left(\mathrm{A}^{*}\right)$ and upper semicontinuity of $\sum_{i} u_{i}$. Indeed, Condition $\left(\mathrm{A}^{*}\right)$ is stronger than payoff security, while upper semicontinuity of $\sum_{i} u_{i}$ implies reciprocal upper semicontinuity of $G$. Since payoff security and reciprocal upper semicontinuity imply better-reply security of $\bar{G}$ (Corollary 5.2 in [22]), we conclude that Condition 6 is satisfied.

Next, choose a sequence $\left(\delta^{n}\right)$ with $\delta^{n} \in(0,1)$ and $\delta^{n} \rightarrow 0$. Since $G$ satisfies Condition $\left(\mathrm{A}^{*}\right)$, we can choose $\nu \in \widehat{\Delta}(X)$ and, for each $i$, a countable set $Q_{i} \subseteq X_{i}$ satisfying the conclusion in Lemma 3 . We will show that part $(i i)$ of Proposition 2 is satisfied for the sequence $\left(\delta^{n}\right)$, the countable sets $Q_{1}, \ldots, Q_{N}$, and the constant sequence $\left(\nu^{n}\right)$ with $\nu^{n}=\nu$ for all $n$. In the remainder of the proof, we will use $\delta^{n}$ to represent the number $\delta^{n} \in(0,1)$ and the vector $\left(\delta_{1}^{n}, \ldots, \delta_{N}^{n}\right)$ with $\delta_{i}^{n}=\delta^{n}$ for each $i$.

To begin, fix $n$ and let

$$
\left(\Delta\left(X_{i}(m)\right), u_{i}^{\left(\delta^{n}, \sigma(m)\right)}\right)_{m \geq 1}
$$


be a sequence where for each $i,\left(X_{i}(m)\right)_{m \geq 1}$ is an increasing sequence of finite subsets of $X_{i}, X_{i}^{n}(m)$ is the finite support of $\sigma_{i}^{n}(m)$,

$$
\bigcup_{m=1}^{\infty} X_{i}^{n}(m) \supseteq Q_{i} \text {, }
$$

$X_{i}^{n}(m) \underset{m \rightarrow \infty}{\longrightarrow} X_{i}$, and $\sigma^{n}(m) \underset{m \rightarrow \infty}{\longrightarrow} \nu$. It suffices to show that $\left(\Delta\left(X_{i}\right), u_{i}^{\left(\delta^{n}, \nu\right)}\right)$ satisfies Condition 5 with respect to the sequence in (4).

To that end, suppose that $\left(\Delta\left(X_{i}(k)\right), u_{i}^{\left(\delta^{n}, \sigma(k)\right)}\right)_{k \geq 1}$ is a subsequence of $\left(\Delta\left(X_{i}(m)\right), u_{i}^{\left(\delta^{n}, \sigma(m)\right)}\right)_{m \geq 1}$ and that $\left(\mu^{k}, u^{\left(\delta^{n}, \sigma(k)\right)}\left(\mu^{k}\right)\right) \in \Delta(X) \times \mathbb{R}^{N}$ is a convergent sequence with limit $(\mu, \gamma) \in \Delta(X) \times \mathbb{R}^{N}$ satisfying $\mu_{i}^{k} \in \Delta\left(X_{i}(k)\right)$ for each $k$ and each $i$. Suppose that $\mu$ is not a Nash equilibrium of $\left(\Delta\left(X_{i}\right), u_{i}^{\left(\delta^{n}, \nu\right)}\right)$. We need to show that there exist $i, \eta>\gamma_{i}$, a subsequence $\left(\mu^{l}\right)$ of $\left(\mu^{k}\right)$ and a sequence $\left(p_{i}^{l}\right)$ such that, for each $l, p_{i}^{l} \in \Delta\left(X_{i}(l)\right)$ and $u_{i}^{\left(\delta^{n}, \nu\right)}\left(p_{i}^{l}, \mu_{-i}^{l}\right) \geq \eta$ and we consider two cases.

Case 1: Suppose that $u_{j}^{\left(\delta^{n}, \nu\right)}(\mu)=\gamma_{j}$ for each player $j$. Since $\mu$ is not a Nash equilibrium of $\left(\Delta\left(X_{i}\right), u_{i}^{\left(\delta^{n}, \nu\right)}\right)$, there exist $i$ and $p_{i} \in \Delta\left(X_{i}\right)$ such that $u_{i}^{\left(\delta^{n}, \nu\right)}\left(p_{i}, \mu_{-i}\right)>u_{i}^{\left(\delta^{n}, \nu\right)}(\mu)$. Choose a positive integer $M$ and a real number $\eta$ so that

$$
u_{i}^{\left(\delta^{n}, \nu\right)}\left(p_{i}, \mu_{-i}\right)-\frac{2}{M} \geq \eta>u_{i}^{\left(\delta^{n}, \nu\right)}(\mu)=\gamma_{i}
$$

Define

$$
\sigma=\left(\sigma_{1}, \ldots, \sigma_{N}\right):=\left(\left(1-\delta^{n}\right) p_{i}+\delta^{n} \nu_{i},\left(1-\delta^{n}\right) \mu_{-i}+\delta^{n} \nu_{-i}\right)
$$

and note that $\sigma_{i} \in \Delta\left(X_{i}, \delta^{n} \nu_{i}\right)$ for each $i$ and that

$$
u_{i}^{\left(\delta^{n}, \nu\right)}\left(p_{i}, \mu_{-i}\right)=u_{i}\left(\left(1-\delta^{n}\right) p_{i}+\delta^{n} \nu_{i},\left(1-\delta^{n}\right) \mu_{-i}+\delta^{n} \nu_{-i}\right)=u_{i}(\sigma) .
$$

Applying Lemma 3, there exist a neighborhood $O_{\sigma_{-i}}$ of $\sigma_{-i}=\left(1-\delta^{n}\right) \mu_{-i}+$ $\delta^{n} \nu_{-i}$ and $\varrho_{i} \in \Delta\left(X_{i}\right)$ with $\varrho_{i}\left(Q_{i}\right)=1$ such that

$$
u_{i}\left(\left(1-\delta^{n}\right) \varrho_{i}+\delta^{n} \nu_{i}, p_{-i}\right)>u_{i}^{\left(\delta^{n}, \nu\right)}\left(p_{i}, \mu_{-i}\right)-\frac{1}{M}, \quad \text { for all } p_{-i} \in O_{\sigma_{-i}} .
$$

Therefore, there exists a neighborhood $O_{\mu_{-i}}$ of $\mu_{-i}$ such that

$$
\begin{aligned}
u_{i}^{\left(\delta^{n}, \nu\right)}\left(\varrho_{i}, q_{-i}\right) & =u_{i}\left(\left(1-\delta^{n}\right) \varrho_{i}+\delta^{n} \nu_{i},\left(1-\delta^{n}\right) q_{-i}+\delta^{n} \nu_{-i}\right) \\
& >u_{i}^{\left(\delta^{n}, \nu\right)}\left(p_{i}, \mu_{-i}\right)-\frac{1}{M}, \quad \text { for all } q_{-i} \in O_{\mu_{-i}} .
\end{aligned}
$$


Now let $\Delta\left(Q_{i}\right)$ and $\Delta^{f}\left(Q_{i}\right)$ denote, respectively, the set of $\theta_{i} \in \Delta\left(X_{i}\right)$ with $\theta_{i}\left(Q_{i}\right)=1$ and the set of $\theta_{i} \in \Delta\left(Q_{i}\right)$ with finite support. Since $u_{i}^{\left(\delta^{n}, \nu\right)}$ is bounded, there exists for every $\epsilon>0$ a measure $\lambda_{i} \in \Delta^{f}\left(Q_{i}\right)$ such that

$$
\left|u_{i}^{\left(\delta^{n}, \nu\right)}\left(\lambda_{i}, q_{-i}\right)-u_{i}^{\left(\delta^{n}, \nu\right)}\left(\varrho_{i}, q_{-i}\right)\right|<\epsilon, \quad \text { for all } q_{-i} \in O_{\mu_{-i}} .
$$

To see this, let $K$ be a bound for $u_{i}^{\left(\delta^{n}, \nu\right)}$. Because $Q_{i}$ is countable, there exist sequences $\left(A_{i}^{k}\right)$ and $\left(\lambda_{i}^{k}\right)$ such that each $A_{i}^{k}$ is a finite subset of $Q_{i}$, each $\lambda_{i}^{k}$ is an element of $\Delta^{f}\left(Q_{i}\right), \varrho_{i}\left(Q_{i} \backslash A_{i}^{k}\right) K \rightarrow 0$, and $\lambda_{i}^{k}\left(B_{i}\right)=\varrho_{i}\left(B_{i}\right)$ for every $B_{i} \subseteq A_{i}^{k}$. Given $k$ and $q_{-i} \in O_{\mu_{-i}}$, we have

$$
\begin{aligned}
& \left|u_{i}^{\left(\delta^{n}, \nu\right)}\left(\lambda_{i}^{k}, q_{-i}\right)-u_{i}^{\left(\delta^{n}, \nu\right)}\left(\varrho_{i}, q_{-i}\right)\right| \\
& =\mid u_{i}\left(\left(1-\delta^{n}\right) \lambda_{i}^{k}+\delta^{n} \nu_{i},\left(1-\delta^{n}\right) q_{-i}+\delta^{n} \nu_{-i}\right) \\
& \quad-u_{i}\left(\left(1-\delta^{n}\right) \varrho_{i}+\delta^{n} \nu_{i},\left(1-\delta^{n}\right) q_{-i}+\delta^{n} \nu_{-i}\right) \mid \\
& =\mid \sum_{a_{i} \in A_{i}^{k}} \lambda_{i}^{k}\left(a_{i}\right) u_{i}\left(\left(1-\delta^{n}\right) a_{i}+\delta^{n} \nu_{i},\left(1-\delta^{n}\right) q_{-i}+\delta^{n} \nu_{-i}\right) \\
& +\sum_{a_{i} \in Q_{i} \backslash A_{i}^{k}} \lambda_{i}^{k}\left(a_{i}\right) u_{i}\left(\left(1-\delta^{n}\right) a_{i}+\delta^{n} \nu_{i},\left(1-\delta^{n}\right) q_{-i}+\delta^{n} \nu_{-i}\right) \\
& -\sum_{a_{i} \in A_{i}^{k}} \varrho_{i}\left(a_{i}\right) u_{i}\left(\left(1-\delta^{n}\right) a_{i}+\delta^{n} \nu_{i},\left(1-\delta^{n}\right) q_{-i}+\delta^{n} \nu_{-i}\right) \\
& -\sum_{a_{i} \in Q_{i} \backslash A_{i}^{k}} \varrho_{i}\left(a_{i}\right) u_{i}\left(\left(1-\delta^{n}\right) a_{i}+\delta^{n} \nu_{i},\left(1-\delta^{n}\right) q_{-i}+\delta^{n} \nu_{-i}\right) \mid .
\end{aligned}
$$

Because $\varrho_{i}\left(Q_{i} \backslash A_{i}^{k}\right) K \rightarrow 0$ and $\lambda_{i}^{k}\left(B_{i}\right)=\varrho_{i}\left(B_{i}\right)$ for every $B_{i} \subseteq A_{i}^{k}$, there is a sufficiently large $k$ such that for every $q_{-i} \in O_{\mu_{-i}}$ we have $\mid u_{i}^{\left(\delta^{n}, \nu\right)}\left(\lambda_{i}^{k}, q_{-i}\right)-$ $u_{i}^{\left(\delta^{n}, \nu\right)}\left(\varrho_{i}, q_{-i}\right) \mid<\epsilon$.

From (7), (5), and (6), we see that there exists a $\lambda_{i} \in \Delta^{f}\left(Q_{i}\right)$ and neighborhood $O_{\mu_{-i}}$ of $\mu_{-i}$ such that

$$
u_{i}^{\left(\delta^{n}, \nu\right)}\left(\lambda_{i}, q_{-i}\right)>u_{i}^{\left(\delta^{n}, \nu\right)}\left(p_{i}, \mu_{-i}\right)-\frac{2}{M} \geq \eta>\gamma_{i}, \quad \text { for all } q_{-i} \in O_{\mu_{-i}} .
$$

Recall that $\left(X_{i}(k)\right)$ is an increasing sequence of finite subsets of $X_{i}$ with $\bigcup_{k} X_{i}(k) \supseteq Q_{i}$. Since $\lambda_{i} \in \Delta^{f}\left(Q_{i}\right)$ and $\mu^{k} \rightarrow \mu$, it follows that there exists a positive integer $L$ such that $\lambda_{i} \in \Delta\left(X_{i}(k)\right)$ and $\mu_{-i}^{k} \in O_{\mu_{-i}}$ for all $k \geq L$. Summarizing, we conclude that $\lambda_{i} \in \Delta\left(X_{i}(k)\right)$ and $u_{i}^{\left(\delta^{n}, \nu\right)}\left(\lambda_{i}, \mu_{-i}^{k}\right) \geq \eta>\gamma_{i}$ for each $k \geq L$. 
Case 2: Suppose that $u_{j}^{\left(\delta^{n}, \nu\right)}(\mu) \neq \gamma_{j}$ for some $j$. Since $\mu^{k} \rightarrow \mu$ and $\sigma(k) \rightarrow \nu$, it follows that

$$
\left(1-\delta^{n}\right) \mu^{k}+\delta^{n} \sigma(k) \rightarrow\left(1-\delta^{n}\right) \mu+\delta^{n} \nu
$$

Since $u^{\left(\delta^{n}, \sigma(k)\right)}\left(\mu^{k}\right) \rightarrow \gamma$ and $u^{\left(\delta^{n}, \sigma(k)\right)}\left(\mu^{k}\right)=u\left(\left(1-\delta^{n}\right) \mu^{k}+\delta^{n} \sigma(k)\right)$, it follows that

$$
u\left(\left(1-\delta^{n}\right) \mu^{k}+\delta^{n} \sigma(k)\right) \rightarrow \gamma .
$$

Combining these two observations, we conclude that $\left(\left(1-\delta^{n}\right) \mu+\delta^{n} \nu, \gamma\right)$ belongs to the closure of the graph of $u$. Since $u_{j}^{\left(\delta^{n}, \nu\right)}(\mu) \neq \gamma_{j}$, it follows that $u_{j}\left(\left(1-\delta^{n}\right) \mu+\delta^{n} \nu\right) \neq \gamma_{j}$ for some $j$ so applying Proposition 5.1 in Reny [22] we see that $u_{i}^{\left(\delta^{n}, \nu\right)}(\mu)=u_{i}\left(\left(1-\delta^{n}\right) \mu+\delta^{n} \nu\right)>\gamma_{i}$ for some $i$.

Now choose a positive integer $M$ and a real number $\eta$ so that

$$
u_{i}^{\left(\delta^{n}, \nu\right)}\left(\mu_{i}, \mu_{-i}\right)-\frac{2}{M} \geq \eta>\gamma_{i}
$$

and duplicate the argument of part 1 with $\mu_{i}$ replacing $p_{i}$.

It would be useful to identify conditions that are satisfied in a reasonably wide class of games arising in economic applications. It can be shown that the combination of two independent conditions, generic entire payoff security and generic local equi-upper semicontinuity, introduced in Carbonell-Nicolau [11], imply a weaker version of Condition $\left(\mathrm{A}^{*}\right)$ which is still strong enough to guarantee the existence of strong limit-of-finite perfect equilibria. This observation, together with Theorem 5, yields the following result. For details, the reader is referred to Carbonell-Nicolau and McLean [12].

Theorem 6. Suppose that $G=\left(X_{i}, u_{i}\right)$ is a compact, metric, Borel game satisfying generic entire payoff security and generic local equi-upper semicontinuity. Suppose further that $\sum_{i} u_{i}$ is upper semicontinuous. Then $G$ possesses a strong limit-of-finite perfect equilibrium, which is also tremblinghand perfect.

Remark 4. Theorem 6 generalizes Corollary 1 in Carbonell-Nicolau [9].

Remark 5. Generic entire payoff security and generic local equi-upper semicontinuity are met in various economic games, as illustrated in CarbonellNicolau (([9], Section 3), ([11], Section 4)).

The following corollary follows immediately from Theorem 6 .

Corollary 4. Suppose that $G=\left(X_{i}, u_{i}\right)$ is a compact, metric, Borel game. Suppose further that each $u_{i}$ is continuous. Then $G$ possesses a strong limitof-finite perfect equilibrium, which is also trembling-hand perfect. 


\subsection{On strong limit-of-finite perfect equilibrium and limit admissibility}

We now turn to the relationship between strong lof perfection and limit admissibility. Unlike Simon and Stinchcombe's [26] lof perfection, strong lof perfection satisfies limit admissibility in continuous games. In fact, for continuous games, the statement of Theorems 5 and 6 can be strengthened as follows:

Theorem 7. Suppose that $G=\left(X_{i}, u_{i}\right)$ is a compact, metric, Borel game. Suppose further that each $u_{i}$ is continuous. Then $G$ possesses a strong limitof-finite perfect equilibrium, and all strong limit-of-finite perfect equilibria are trembling-hand perfect.

Proof. That $G$ possesses a strong lof perfect equilibrium follows immediately from Theorem 5. To see that all strong lof perfect equilibria are thp, let $\mu$ be a strong lof perfect equilibrium. Then there is a sequence $\left(\eta^{n}\right)$ with $\eta^{n} \rightarrow 0$ such that for every sequence $\left(\varepsilon^{n}\right)$ with $0<\varepsilon^{n} \rightarrow 0$, there exists a finite $\left(\varepsilon^{n}\right)$ approximation $\left(\bar{G}_{\left(Y^{n}, \xi^{n}\right)}^{\eta^{n}}\right)$ of $\left(\bar{G}_{\eta^{n}}\right)$ such that each $\bar{G}_{\left(Y^{n}, \xi^{n}\right)}^{\eta^{n}}$ possesses a Nash equilibrium $\mu^{n}$ and $\mu^{n} \rightarrow \mu$.

For each $i$, let $K\left(\Delta\left(X_{i}\right)\right)$ be the set of nonempty, compact, convex subsets of $\Delta\left(X_{i}\right)$, endowed with the Hausdorff metric induced by the Prokhorov metric on $\Delta\left(X_{i}\right)$. Set $K(\Delta(X)):=\times_{i=1}^{N} K\left(\Delta\left(X_{i}\right)\right)$. Let $\mathcal{C}_{i}$ be the set of continuous maps $f_{i}: \Delta(X) \rightarrow \mathbb{R}$ such that the map $\sigma_{i} \mapsto f_{i}\left(\sigma_{i}, \sigma_{-i}\right)$ defined on $\Delta\left(X_{i}\right)$ is quasiconcave for every $\sigma_{-i} \in \times_{j \neq i} \Delta\left(X_{j}\right)$. Let $\mathcal{C}:=\times_{i=1}^{N} \mathcal{C}_{i}$. Endow the set $\mathcal{C}$ with the following metric $d: \mathcal{C} \times \mathcal{C} \rightarrow \mathbb{R}$ :

$$
d\left(\left(f_{1}, \ldots, f_{N}\right),\left(g_{1}, \ldots, g_{N}\right)\right):=\sum_{i=1}^{N} \sup _{\sigma \in \Delta(X)}\left|f_{i}(\sigma)-g_{i}(\sigma)\right| .
$$

The correspondence

$$
\mathcal{E}: K(\Delta(X)) \times \mathcal{C} \rightrightarrows \Delta(X)
$$

assigns to each tuple

$$
(\Sigma, f)=\left(\left(\Sigma_{1}, \ldots, \Sigma_{N}\right),\left(f_{1}, \ldots, f_{N}\right)\right)
$$

in $K(\Delta(X)) \times \mathcal{C}$ the set $\mathcal{E}(\Sigma, f)$ of Nash equilibria of the game $\left(\Sigma_{i}, f_{i}\right)_{i=1}^{N}$. The correspondence $\mathcal{E}$ is nonempty-valued and (by Theorem 3.4 of $\mathrm{Yu}$ [27]) upper hemicontinuous. Consequently, for each $n$,

$$
\mathcal{E}\left(\Delta\left(X, \eta^{n}\right), u\right) \neq \emptyset,
$$


where

$$
\left(\Delta\left(X, \eta^{n}\right), u\right):=\left(\left(\Delta\left(X_{1}, \eta_{1}^{n}\right), \ldots, \Delta\left(X_{N}, \eta_{N}^{n}\right)\right),\left(u_{1}, \ldots, u_{N}\right)\right)
$$

and $\mathcal{E}$ is upper hemicontinuous at $\left(\Delta\left(X, \eta^{n}\right), u\right)$. Because $\mathcal{E}$ is upper hemicontinuous at $\left(\Delta\left(X, \eta^{n}\right), u\right)$ for each $n$, there exists, for each $n$, a neighborhood $V_{\left(\Delta\left(X, \eta^{n}\right), u\right)}$ of $\left(\Delta\left(X, \eta^{n}\right), u\right)$ such that

$$
(\Sigma, f) \in V_{\left(\Delta\left(X, \eta^{n}\right), u\right)} \Rightarrow \mathcal{E}(\Sigma, f) \subseteq N_{\frac{1}{n}}\left(\mathcal{E}\left(\Delta\left(X, \eta^{n}\right), u\right)\right) .
$$

For each $n$, we now prove the following:

Claim. If $\left(\bar{G}_{\left(Y^{m}, \xi^{m}\right)}^{\eta^{n}}\right)$ is a sequence such that each $\bar{G}_{\left(Y^{m}, \xi^{m}\right)}^{\eta^{n}}$ is a finite $\frac{1}{m}$ approximation of $\bar{G}_{\eta^{n}}$, then

$$
\Delta\left(Y^{m}, \xi^{m}\right) \rightarrow \Delta\left(X, \eta^{n}\right)
$$

i.e., the sequence of sets $\left(\Delta\left(Y^{m}, \xi^{m}\right)\right)$ is Hausdorff convergent with limit $\Delta\left(X, \eta^{n}\right)$.

Proof of Claim. Fix $n$ and let $\left(\bar{G}_{\left(Y^{m}, \xi^{m}\right)}^{\eta^{n}}\right)$ be a sequence such that each $\bar{G}_{\left(Y^{m}, \xi^{m}\right)}^{\eta^{n}}$ is a finite $\frac{1}{m}$-approximation of $\bar{G}_{\eta^{n}}$. Combining Theorems 5.1.11 and 5.2.10 in Beer [7], it suffices to show that

$$
\operatorname{Ls}\left(\Delta\left(Y^{m}, \xi^{m}\right)\right) \subseteq \Delta\left(X, \eta^{n}\right) \subseteq \operatorname{Li}\left(\Delta\left(Y^{m}, \xi^{m}\right)\right)
$$

Suppose that $\rho \in \operatorname{Ls}\left(\Delta\left(Y^{m}, \xi^{m}\right)\right)$. Then there exist a subsequence $\left(m_{k}\right)$ of $(m)$ and a sequence $\left(\rho^{m_{k}}\right)$ such that $\rho^{m_{k}} \in \Delta\left(Y^{m_{k}}, \xi^{m_{k}}\right)$ for each $k$ and $\rho^{m_{k}} \rightarrow$ $\rho$. Write $\rho_{i}^{m_{k}}=\theta_{i}^{m_{k}}+\xi_{i}^{m_{k}}$, where $\theta_{i}^{m_{k}}:=\rho_{i}^{m_{k}}-\xi_{i}^{m_{k}}$. Since $1>\xi_{i}^{m_{k}}\left(Y_{i}^{m_{k}}\right)>0$, $\theta_{i}^{m_{k}}\left(Y_{i}^{m_{k}}\right)=\rho_{i}^{m_{k}}\left(Y_{i}^{m_{k}}\right)-\xi_{i}^{m_{k}}\left(Y_{i}^{m_{k}}\right)=1-\xi_{i}^{m_{k}}\left(Y_{i}^{m_{k}}\right)>0$, and we have

$$
\rho_{i}^{m_{k}}=\left(1-\xi_{i}^{m_{k}}\left(Y_{i}^{m_{k}}\right)\right) \frac{\theta_{i}^{m_{k}}}{\theta_{i}^{m_{k}}\left(Y_{i}^{m_{k}}\right)}+\xi_{i}^{m_{k}}\left(Y_{i}^{m_{k}}\right) \frac{\xi_{i}^{m_{k}}}{\xi_{i}^{m_{k}}\left(Y_{i}^{m_{k}}\right)} .
$$

Hence, because there exists $\theta_{i} \in \Delta\left(X_{i}\right)$ such that $\frac{\theta_{i}^{m_{k}}}{\theta_{i}^{m}\left(Y_{i}^{m_{k}}\right)} \rightarrow \theta_{i}$ (passing to a subsequence if necessary) and because $\xi_{i}^{m_{k}}\left(Y_{i}^{m_{k}}\right) \rightarrow \eta_{i}^{n}\left(X_{i}\right)$ and $\frac{\xi_{i}^{m_{k}}}{\xi_{i}^{m_{k}}\left(Y_{i}^{m_{k}}\right)} \rightarrow$ $\frac{\eta_{i}^{n}}{\eta_{i}^{n}\left(X_{i}\right)}$, we see that

$$
\rho_{i}^{m_{k}} \rightarrow\left(1-\eta_{i}^{n}\left(X_{i}\right)\right) \theta_{i}+\eta_{i}^{n}\left(X_{i}\right) \frac{\eta_{i}^{n}}{\eta_{i}^{n}\left(X_{i}\right)}
$$


Consequently, $\rho_{i}=\left(1-\eta_{i}^{n}\left(X_{i}\right)\right) \theta_{i}+\eta_{i}^{n}\left(X_{i}\right) \frac{\eta_{i}^{n}}{\eta_{i}^{n}\left(X_{i}\right)}$ for each $i$, and so $\rho \in$ $\Delta\left(X, \eta^{n}\right)$. This establishes the inclusion $\operatorname{Ls}\left(\Delta\left(Y^{m}, \xi^{m}\right)\right) \subseteq \Delta\left(X, \eta^{n}\right)$.

Now suppose that $\rho \in \Delta\left(X, \eta^{n}\right)$ and for each $i$, define $\lambda_{i}:=\rho_{i}-\eta_{i}^{n}$. Since $\lambda_{i}\left(X_{i}\right)=1-\eta_{i}^{n}\left(X_{i}\right)>0$, we have

$$
\rho_{i}=\left(1-\eta_{i}^{n}\left(X_{i}\right)\right) \frac{\lambda_{i}}{\lambda_{i}\left(X_{i}\right)}+\eta_{i}^{n}\left(X_{i}\right) \frac{\eta_{i}^{n}}{\eta_{i}^{n}\left(X_{i}\right)} .
$$

Because the set of finitely supported measures in $\Delta\left(X_{i}\right)$ is dense in $\Delta\left(X_{i}\right)$, there exists a sequence $\left(\lambda_{i}^{k}\right)$ of finitely supported measures in $\Delta\left(X_{i}\right)$ such that $\lambda_{i}^{k} \rightarrow \frac{\lambda_{i}}{\lambda_{i}\left(X_{i}\right)}$. Since $Y_{i}^{m} \rightarrow X_{i}$ and since each $\lambda_{i}^{k}$ has finite support, for each $k$ and for every sufficiently large $m$, there exists $\widehat{\lambda}_{i} \in \Delta\left(X_{i}\right)$ with support in $Y_{i}^{m}$ such that $\pi\left(\lambda_{i}^{k}, \widehat{\lambda}_{i}\right)<\frac{1}{k} \cdot{ }^{7}$

\footnotetext{
${ }^{7}$ Given $k$, let $\left\{a_{1}, \ldots, a_{\ell}\right\}$ be the finite support of $\lambda_{i}^{k}$. Since $Y_{i}^{m} \rightarrow X_{i}$, for each $l \in$ $\{1, \ldots, \ell\}$ one can choose a sequence $\left(\alpha_{l}^{m}\right)$ such that $\alpha_{l}^{m} \in Y_{i}^{m}$ for each $m$ and $\alpha_{l}^{m} \underset{m \rightarrow \infty}{\longrightarrow} a_{l}$. For each $m$, define $\widehat{\nu}_{i}^{m}$ in $\Delta\left(X_{i}\right)$ by

$$
\widehat{\nu}_{i}^{m}\left(\left\{\alpha_{l}^{m}\right\}\right):=\lambda_{i}^{k}\left(\left\{a_{l}\right\}\right), \quad \text { for each } l \in\{1, \ldots, \ell\}
$$

and note that $\operatorname{supp}\left(\widehat{\nu}_{i}^{m}\right)=\left\{\alpha_{1}^{m}, . ., \alpha_{\ell}^{m}\right\} \subseteq Y_{i}^{m}$ for each $m$. Let $\widetilde{\nu}_{i}^{m} \in \Delta\left(X_{i}\right)$ be defined by $\widetilde{\nu}_{i}^{m}\left(\left\{y_{i}^{m}\right\}\right):=\frac{1}{\# Y_{i}^{m}}$ for each $y_{i}^{m} \in Y_{i}^{m}$, where $\# Y_{i}^{m}$ denotes the cardinality of the finite set $Y_{i}^{m}$. Define, for each $m$,

$$
\nu_{i}^{m}:=\left(1-\frac{1}{m}\right) \widehat{\nu}_{i}^{m}+\frac{1}{m} \widetilde{\nu}_{i}^{m}
$$
}

and note that $\operatorname{supp}\left(\nu_{i}^{m}\right)=Y_{i}^{m}$ for each $m$. To see that $\nu_{i}^{m} \rightarrow \lambda_{i}^{k}$, let $O_{i}$ be an open set in $X_{i}$ and define $I:=\left\{l: a_{l} \in O_{i}\right\}$. Then there exists an $\widehat{m}$ such that, $\alpha_{l}^{m} \in O_{i}$ for each $m>\widehat{m}$ and each $l \in I$. Therefore, $m>\widehat{m}$ implies that

$$
\lambda_{i}^{k}\left(O_{i} \cap\left\{a_{1}, \ldots, a_{\ell}\right\}\right)=\sum_{l \in I} \lambda_{i}^{k}\left(\left\{a_{l}\right\}\right)=\sum_{l \in I} \widehat{\nu}_{i}^{m}\left(\left\{\alpha_{l}^{m}\right\}\right) \leq \widehat{\nu}_{i}^{m}\left(O_{i} \cap\left\{\alpha_{1}^{m}, \ldots, \alpha_{\ell}^{m}\right\}\right),
$$

so for $m>\widehat{m}$, we have

$$
\begin{aligned}
\nu_{i}^{m}\left(O_{i}\right) & =\left(1-\frac{1}{m}\right) \widehat{\nu}_{i}^{m}\left(O_{i}\right)+\frac{1}{m} \widetilde{\nu}_{i}^{m}\left(O_{i}\right) \\
& =\widehat{\nu}_{i}^{m}\left(O_{i}\right)+\frac{1}{m}\left(\widetilde{\nu}_{i}^{m}\left(O_{i}\right)-\widehat{\nu}_{i}^{m}\left(O_{i}\right)\right) \\
& \geq \widehat{\nu}_{i}^{m}\left(O_{i}\right)-\frac{1}{m} \\
& =\widehat{\nu}_{i}^{m}\left(O_{i} \cap\left\{\alpha_{1}^{m}, \ldots, \alpha_{\ell}^{m}\right\}\right)-\frac{1}{m} \\
& \geq \lambda_{i}^{k}\left(O_{i} \cap\left\{a_{1}, \ldots, a_{\ell}\right\}\right)-\frac{1}{m} \\
& =\lambda_{i}^{k}\left(O_{i}\right)-\frac{1}{m},
\end{aligned}
$$

from which it follows that $\underline{\lim } \nu_{i}^{m}\left(O_{i}\right) \geq \lambda_{i}^{k}\left(O_{i}\right)$. 
Since $\lambda_{i}^{k} \rightarrow \frac{\lambda_{i}}{\lambda_{i}\left(X_{i}\right)}$, this implies that there exists a sequence $\left(\widehat{\lambda}_{i}^{m}\right)$ such that $\widehat{\lambda}_{i}^{m} \in \Delta\left(X_{i}\right)$ has support in $Y_{i}^{m}$ for each $k$ and $\widehat{\lambda}_{i}^{m} \rightarrow \frac{\lambda_{i}}{\lambda_{i}\left(X_{i}\right)}$. In light of (11), therefore, we have

$$
\left(1-\xi_{i}^{m}\left(Y_{i}^{m}\right)\right) \widehat{\lambda}_{i}^{m}+\xi_{i}^{m}\left(Y_{i}^{m}\right) \frac{\xi_{i}^{m}}{\xi_{i}^{m}\left(Y_{i}^{m}\right)} \rightarrow \rho_{i}
$$

(recall that $\xi_{i}^{m}\left(Y_{i}^{m}\right) \rightarrow \eta_{i}^{n}\left(X_{i}\right)$ and $\left.\frac{\xi_{i}^{m}}{\xi_{i}^{m}\left(Y_{i}^{m}\right)} \rightarrow \frac{\eta_{i}^{n}}{\eta_{i}^{n}\left(X_{i}\right)}\right)$. For each $n$, the measure

$$
\left(1-\xi_{i}^{m}\left(Y_{i}^{m}\right)\right) \widehat{\lambda}_{i}^{m}+\xi_{i}^{m}\left(Y_{i}^{m}\right) \frac{\xi_{i}^{m}}{\xi_{i}^{m}\left(Y_{i}^{m}\right)}
$$

is clearly a member of $\Delta\left(Y_{i}^{m}, \xi_{i}^{m}\right)$. We conclude that for each $i$ there exists a sequence $\left(v_{i}^{m}\right)$ such that $v_{i}^{m} \in \Delta\left(Y_{i}^{m}, \xi_{i}^{m}\right)$ for each $m$ and $v_{i}^{m} \rightarrow \rho_{i}$. Hence $\rho \in \operatorname{Li}\left(\Delta\left(Y^{m}, \xi^{m}\right)\right)$. This establishes (10) and the proof of the Claim is complete.

Applying the claim, we conclude that, for each $n$, there exists a $\delta_{n}^{*}>$ 0 such that $(\Delta(Y, \xi), u) \in N_{\varepsilon^{n}}\left(\Delta\left(X, \eta^{n}\right), u\right)$ whenever $\bar{G}_{(Y, \xi)}^{\eta^{n}}$ is a finite $\delta$ approximation of $\bar{G}_{\eta^{n}}$ satisfying $0<\delta<\delta_{n}^{*}$. Choose a sequence $\alpha^{n} \rightarrow 0$ satisfying $0<\alpha^{n}<\delta_{n}^{*}$ for each $n$. Since $\mu$ is a strong lof perfect equilibrium, there is a finite $\left(\alpha^{n}\right)$-approximation $\left(\bar{G}_{(Y, \xi)}^{\eta^{n}}\right)$ of $\left(\bar{G}_{\eta^{n}}\right)$ such that each $\bar{G}_{(Y, \xi)}^{\eta^{n}}$ possesses a Nash equilibrium $\mu^{n}$ and $\mu^{n} \rightarrow \mu$. From the definition of the sequence $\left(\alpha^{n}\right)$, it follows that

$$
\left(\Delta\left(Y^{n}, \xi^{n}\right), u\right) \in N_{\varepsilon^{n}}\left(\Delta\left(X, \eta^{n}\right), u\right) \subseteq V_{\left(\Delta\left(X, \eta^{n}\right), u\right)},
$$

where

$$
\left(\Delta\left(Y^{n}, \xi^{n}\right), u\right):=\left(\left(\Delta\left(Y_{1}^{n}, \xi_{1}^{n}\right), \ldots, \Delta\left(Y_{N}^{n}, \xi_{N}^{n}\right)\right),\left(u_{1}, \ldots, u_{N}\right)\right) .
$$

Consequently, $\mu^{n} \in \mathcal{E}\left(\Delta\left(Y^{n}, \xi^{n}\right), u\right)$ for each $n$, and in light of (12) and (8), we obtain

$$
\mu^{n} \in N_{\frac{1}{n}}\left(\mathcal{E}\left(\Delta\left(X, \eta^{n}\right), u\right)\right) .
$$

This, together with the fact that $\mu^{n} \rightarrow \mu$, implies that there exists a sequence $\left(\sigma^{n}\right)$ such that $\sigma^{n} \in \mathcal{E}\left(\Delta\left(X, \eta^{n}\right), u\right)$ for each $n$ and $\sigma^{n} \rightarrow \mu$. In other words, $\mu$ is a thp profile. This completes the proof.

Remark 6. Example 2 in Bajoori et al. [5] shows that there are continuous games that possess thp equilibria that fail to be lof perfect. Since strong lof perfection strengthens lof perfection (Proposition 1), there are thp equilibria that are not strong lof perfect. 
It is shown in Simon and Stinchcombe [26] that any trembling-hand perfect equilibrium of a compact, metric, Borel game $G=\left(X_{i}, u_{i}\right)$ with each $u_{i}$ continuous is limit admissible. From this observation and Theorem 7, we obtain the following result.

Theorem 8. Suppose that $G=\left(X_{i}, u_{i}\right)$ is a compact, metric, Borel game. Suppose further that each $u_{i}$ is continuous. Then any strong limit-of-finite perfect equilibrium of $G$ is limit admissible.

\section{References}

[1] Aliprantis C. D., and K. C. Border (2006), Infinite Dimensional Analysis. Springer, Berlin.

[2] Al-Najjar, N. (1995), "Strategically stable equilibria in games with infinitely many pure strategies," Mathematical Social Sciences, 29, 151164.

[3] Bagh, A., and A. Jofre (2006), "Weak reciprocal upper semi-continuity and Better reply secure games," Econometrica, 74, 1715-1721.

[4] Bagh, A. (2010), "Variational convergence: approximation and existence of equilibria in discontinuous games," Journal of Economic Theory, 145, 1244-1268.

[5] Bajoori, E., J. Flesch, and D. Vermeulen (2011), "Perfect equilibrium in games with compact action spaces," Maastricht University RM/11/029.

[6] Barelli, P., and I. Soza (2010), "On the existence of Nash equilibria in discontinuous and qualitative games," mimeo.

[7] Beer, G. A. (1993), Topologies on Closed and Closed Convex Sets. Kluwer Academic Publishers, Dordrecht.

[8] Billingsley, P. (1968), Convergence of Probability Measures. Wiley, New York.

[9] Carbonell-Nicolau, O. (2011), "The existence of perfect equilibrium in discontinuous games," Games, 2, 235-256. 
[10] Carbonell-Nicolau, O. (2011), "Perfect and limit admissible perfect equilibria in discontinuous games," Journal of Mathematical Economics, 47, 531-540.

[11] Carbonell-Nicolau, O. (2011), "On the existence of pure-strategy perfect equilibrium in discontinuous games," Games and Economic Behavior, $71,23-48$.

[12] Carbonell-Nicolau, O., and R. P. McLean (2012), "Approximation results for discontinuous games with an application to equilibrium refinement," mimeo.

[13] Carmona, G. (2011), "Understanding some recent existence results for discontinuous games," Economic Theory, 48, 31-45.

[14] Carmona, G. (2011), "Symposium on: Existence of Nash equilibria in discontinuous games," Economic Theory, 48, 1-4.

[15] de Castro, L. I. (2011), "Equilibrium existence and approximation of regular discontinuous games," Economic Theory, 48, 67-85.

[16] Dasgupta, P., and E. Maskin (1986), "The existence of equilibrium in discontinuous economic games, I: Theory," Review of Economic Studies $53,1-26$.

[17] Gürkan, G., and J. S. Pang (2009), "Approximizations of Nash equilibria," Mathematical Programming B, 117, 223-253.

[18] Lebrun, B. (1996), "Existence of an equilibrium in first price auctions," Economic Theory, 7, 421-443.

[19] Lucchetti, R, and F. Patrone (1986), "Closure and upper semicontinuity results in mathematical programming, Nash and economic equilibria," Optimization, 17, 619-628.

[20] McLennan, A., Monteiro, P. K., and R. Tourky, "Games with discontinuous payoffs: a strengthening of Reny's existence theorem," Econometrica, 79, 1643-1664.

[21] Prokopovych, P. (2011), "On equilibrium existence in payoff secure games," Economic Theory, 48, 5-16. 
[22] Reny, P. J. (1999), "On the existence of pure and mixed strategy Nash equilibria in discontinuous games," Econometrica, 67, 1029-1056.

[23] Reny, P. J. (2011), "Strategic approximations of discontinuous games," Economic Theory, 48, 17-29.

[24] Selten, R. (1975), "Reexamination of the perfectness concept for equilibrium points in extensive games," International Journal of Game Theory, $4,25-55$.

[25] Simon, L. K. (1987), "Games with discontinuous payoffs," Review of Economic Studies, 54, 569-597.

[26] Simon, L. K., and M. B. Stinchcombe (1995), "Equilibrium refinement for infinite normal-form games," Econometrica, 63, 1421-1443.

[27] Yu, J. (1999), "Essential equilibria of $n$-person noncooperative games," Journal of Mathematical Economics, 31, 361-372. 